\title{
Parameterized Approximation Algorithms for some Location Problems in Graphs
}

\author{
Arne Leitert and Feodor F. Dragan \\ Department of Computer Science, \\ Kent State University, Kent, Ohio, USA \\ aleitert@cs.kent.edu, dragan@cs.kent.edu
}

\begin{abstract}
We develop efficient parameterized, with additive error, approximation algorithms for the (Connected) $r$-Domination problem and the (Connected) $p$-Center problem for unweighted and undirected graphs. Given a graph $G$, we show how to construct a (connected) $(r+\mathcal{O}(\mu))$ dominating set $D$ with $|D| \leq\left|D^{*}\right|$ efficiently. Here, $D^{*}$ is a minimum (connected) $r$-dominating set of $G$ and $\mu$ is our graph parameter, which is the tree-breadth or the cluster diameter in a layering partition of $G$. Additionally, we show that a $+\mathcal{O}(\mu)$-approximation for the (Connected) $p$-Center problem on $G$ can be computed in polynomial time. Our interest in these parameters stems from the fact that in many real-world networks, including Internet application networks, web networks, collaboration networks, social networks, biological networks, and others, and in many structured classes of graphs these parameters are small constants.
\end{abstract}

\section{Introduction}

The (Connected) $r$-Domination problem and the (Connected) $p$-Center problem, along with the $p$-Median problem, are among basic facility location problems with many applications in data clustering, network design, operations research to name a few. Let $G=(V, E)$ be an unweighted and undirected graph. Given a radius $r(v) \in \mathbb{N}$ for each vertex $v$ of $G$, indicating within what radius a vertex $v$ wants to be served, the $r$-Domination problem asks to find a set $D \subseteq V$ of minimum cardinality such that $d_{G}(v, D) \leq r(v)$ for every $v \in V$. The Connected $r$-Domination problem asks to find an $r$-dominating set $D$ of minimum cardinality with an additional requirement that $D$ needs to induce a connected subgraph of $G$. When $r(v)=1$ for every $v \in V$, one gets the classical (Connected) Domination problem. Note that the Connected $r$-Domination problem is a natural generalization of the Steiner Tree problem (where each vertex $t$ in the target set has $r(t)=0$ and each other vertex $s$ has $r(s)=\operatorname{diam}(G)$ ). The connectedness of $D$ is important also in network design and analysis applications (e.g. in finding a small backbone of a network). It is easy to see also that finding minimum connected dominating sets is equivalent to finding spanning trees with the maximum possible number of leaves.

The (closely related) $p$-Center problem asks to find in $G$ a set $C \subseteq V$ of at most $p$ vertices such that the value $\max _{v \in V} d_{G}(v, C)$ is minimized. If, additionally, 
$C$ is required to induce a connected subgraph of $G$, then one gets the Connected p-Center problem.

The domination problem is one of the most well-studied NP-hard problems in algorithmic graph theory. To cope with the intractability of this problem it has been studied both in terms of approximability (relaxing the optimality) and fixed-parameter tractability (relaxing the runtime). From the approximability prospective, a logarithmic approximation factor can be found by using a simple greedy algorithm, and finding a sublogarithmic approximation factor is NPhard [19. The problem is in fact Log-APX-complete [14]. The Domination problem is notorious also in the theory of fixed-parameter tractability (see, e. g., [11]18 for an introduction to parameterized complexity). It was the first problem to be shown W[2]-complete [11, and it is hence unlikely to be FPT, i. e., unlikely to have an algorithm with runtime $f(k) n^{c}$ for $f$ a computable function, $k$ the size of an optimal solution, $c$ a constant, and $n$ the number of vertices of the input graph. Similar results are known also for the connected domination problem [17.

The $p$-Center problem is known to be NP-hard on graphs. However, for it, a simple and efficient factor 2 approximation algorithm exists [16]. Furthermore, it is a best possible approximation algorithm in the sense that an approximation with factor less than 2 is proven to be NP-hard (see [16] for more details). The NP-hardness of the Connected $p$-Center problem is shown in [20].

Recently, in [7], a new type of approximability result (call it a parameterized approximability result) was obtained: there exists a polynomial time algorithm which finds in an arbitrary graph $G$ having a minimum $r$-dominating set $D$ a set $D^{\prime}$ such that $\left|D^{\prime}\right| \leq|D|$ and each vertex $v \in V$ is within distance at most $r(v)+2 \delta$ from $D^{\prime}$, where $\delta$ is the hyperbolicity parameter of $G$ (see [7] for details). We call such a $D^{\prime}$ an $(r+2 \delta)$-dominating set of $G$. Later, in [13], this idea was extended to the $p$-Center problem: there is a quasi-linear time algorithm for the $p$-Center problem with an additive error less than or equal to six times the input graph's hyperbolicity (i.e., it finds a set $C^{\prime}$ with at most $p$ vertices such that $\left.\max _{v \in V} d_{G}\left(v, C^{\prime}\right) \leq \min _{C \subseteq V,|C| \leq p} \max _{v \in V} d_{G}(v, C)+6 \delta\right)$. We call such a $C^{\prime}$ a $+6 \delta$-approximation for the $p$-Center problem.

In this paper, we continue the line of research started in [7] and [13. Unfortunately, the results of [7]13] are hardly extendable to connected versions of the $r$-Domination and $p$-Center problems. It remains an open question whether similar approximability results parameterized by the graph's hyperbolicity can be obtained for the Connected $r$-Domination and Connected $p$-Center problems. Instead, we consider two other graph parameters: the tree-breadth $\rho$ and the cluster diameter $\Delta$ in a layering partition (formal definitions will be given in the next sections). Both parameters (like the hyperbolicity) capture the metric tree-likeness of a graph (see, e.g., [2] and papers cited therein). As demonstrated in [2], in many real-world networks, including Internet application networks, web networks, collaboration networks, social networks, biological networks, and others, as well as in many structured classes of graphs the parameters $\delta, \rho$, and $\Delta$ are small constants. 
We show here that, for a given $n$-vertex, $m$-edge graph $G$, having a minimum $r$-dominating set $D$ and a minimum connected $r$-dominating set $C$ :

- an $(r+\Delta)$-dominating set $D^{\prime}$ with $\left|D^{\prime}\right| \leq|D|$ can be computed in linear time;

- a connected $(r+2 \Delta)$-dominating set $C^{\prime}$ with $\left|C^{\prime}\right| \leq|C|$ can be computed in $\mathcal{O}(m \alpha(n) \log \Delta)$ time (where $\alpha(n)$ is the inverse Ackermann function);

- a $+\Delta$-approximation for the $p$-Center problem can be computed in linear time;

- a $+2 \Delta$-approximation for the connected $p$-Center problem can be computed in $\mathcal{O}(m \alpha(n) \log \min (\Delta, p))$ time.

Furthermore, given a tree-decomposition with breadth $\rho$ for $G$ :

- an $(r+\rho)$-dominating set $D^{\prime}$ with $\left|D^{\prime}\right| \leq|D|$ can be computed in $\mathcal{O}(n m)$ time;

- a connected $(r+5 \rho)$-dominating set $C^{\prime}$ with $\left|C^{\prime}\right| \leq|C|$ can be computed in $\mathcal{O}(n m)$ time;

- a $+\rho$-approximation for the $p$-Center problem can be computed in $\mathcal{O}(n m \log n)$ time;

- a $+5 \rho$-approximation for the Connected $p$-Center problem can be computed in $\mathcal{O}(n m \log n)$ time.

To compare these results with the results of [7]13, notice that, for any graph $G$, its hyperbolicity $\delta$ is at most $\Delta[2]$ and at most two times its tree-breadth $\rho[6]$, and the inequalities are sharp.

Note that, for split graphs (graphs in which the vertices can be partitioned into a clique and an independent set), all three parameters are at most 1. Additionally, as shown in [8], there is (under reasonable assumptions) no polynomial-time algorithm to compute a sublogarithmic-factor approximation for the (Connected) Domination problem in split graphs. Hence, there is no such algorithm even for constant $\delta, \rho$, and $\Delta$.

One can extend this result to show that there is no polynomial-time algorithm $\mathcal{A}$ which computes, for any constant $c, \mathrm{a}+c \log n$-approximation for split graphs. Hence, there is no polynomial-time $+c \Delta \log n$-approximation algorithm in general. Consider a given split graph $G=(C \cup I, E)$ with $n$ vertices where $C$ induces a clique and $I$ induces an independent set. Create a graph $H=$ $\left(C_{H} \cup I_{H}, E_{H}\right)$ by, first, making $n$ copies of $G$. Let $C_{H}=C_{1} \cup C_{2} \cup \ldots \cup C_{n}$ and $I_{H}=I_{1} \cup I_{2} \cup \ldots \cup I_{n}$. Second, make the vertices in $C_{H}$ pairwise adjacent. Then, $C_{H}$ induces a clique and $I_{H}$ induces an independent set. If there is such an algorithm $\mathcal{A}$, then $\mathcal{A}$ produces a (connected) dominating set $D_{\mathcal{A}}$ for $H$ which hast at most $2 c \log n$ more vertices that a minimum (connected) dominating set $D$. Thus, by pigeonhole principle, $H$ contains a clique $C_{i}$ for which $\left|C_{i} \cap D_{\mathcal{A}}\right|=\left|C_{i} \cap D\right|$. Therefore, such an algorithm $\mathcal{A}$ would allow to solve the (Connected) Domination problem for split graphs in polynomial time. 


\section{Preliminaries}

All graphs occurring in this paper are connected, finite, unweighted, undirected, without loops, and without multiple edges. For a graph $G=(V, E)$, we use $n=|V|$ and $m=|E|$ to denote the cardinality of the vertex set and the edge set of $G$, respectively.

The length of a path from a vertex $v$ to a vertex $u$ is the number of edges in the path. The distance $d_{G}(u, v)$ in a graph $G$ of two vertices $u$ and $v$ is the length of a shortest path connecting $u$ and $v$. The distance between a vertex $v$ and a set $S \subseteq V$ is defined as $d_{G}(v, S)=\min _{u \in S} d_{G}(u, v)$. For a vertex $v$ of $G$ and some positive integer $r$, the set $N_{G}^{r}[v]=\left\{u \mid d_{G}(u, v) \leq r\right\}$ is called the $r$ neighbourhood of $v$. The eccentricity $\operatorname{ecc}_{G}(v)$ of a vertex $v$ is $\max _{u \in V} d_{G}(u, v)$. For a set $S \subseteq V$, its eccentricity is $\operatorname{ecc}_{G}(S)=\max _{u \in V} d_{G}(u, S)$.

For some function $r: V \rightarrow \mathbb{N}$, a vertex $u$ is $r$-dominated by a vertex $v$ (by a set $S \subseteq V)$, if $d_{G}(u, v) \leq r(u)\left(d_{G}(u, S) \leq r(u)\right.$, respectively). A vertex set $D$ is called an $r$-dominating set of $G$ if each vertex $u \in V$ is $r$ dominated by $D$. Additionally, for some non-negative integer $\phi$, we say a vertex is $(r+\phi)$-dominated by a vertex $v$ (by a set $S \subseteq V)$, if $d_{G}(u, v) \leq r(u)+\phi\left(d_{G}(u, S) \leq r(u)+\phi\right.$, respectively). An $(r+\phi)$-dominating set is defined accordingly. For a given graph $G$ and function $r$, the (Connected) $r$-Domination problem asks for the smallest (connected) vertex set $D$ such that $D$ is an $r$-dominating set of $G$.

The degree of a vertex $v$ is the number of vertices adjacent to it. For a vertex set $S$, let $G[S]$ denote the subgraph of $G$ induced by $S$. A vertex set $S$ is a separator for two vertices $u$ and $v$ in $G$ if each path from $u$ to $v$ contains a vertex $s \in S$; in this case we say $S$ separates $u$ from $v$.

A tree-decomposition of a graph $G=(V, E)$ is a tree $T$ with the vertex set $\mathcal{B}$ where each vertex of $T$, called bag, is a subset of $V$ such that: (i) $V=\bigcup_{B \in \mathcal{B}} B$, (ii) for each edge $u v \in E$, there is a bag $B \in \mathcal{B}$ with $u, v \in B$, and (iii) for each vertex $v \in V$, the bags containing $v$ induce a subtree of $T$. A tree-decomposition $T$

of $G$ has breadth $\rho$ if, for each bag $B$ of $T$, there is a vertex $v$ in $G$ with $B \subseteq N_{G}^{\rho}[v]$. The tree-breadth of a graph $G$ is $\rho$, written as $\operatorname{tb}(G)=\rho$, if $\rho$ is the minimal breadth of all tree-decomposition for $G$. A tree-decomposition $T$ of $G$ has length $\lambda$ if, for each bag $B$ of $T$ and any two vertices $u, v \in B, d_{G}(u, v) \leq \lambda$. The treelength of a graph $G$ is $\lambda$, written as $\operatorname{tl}(G)=\lambda$, if $\lambda$ is the minimal length of all tree-decomposition for $G$.

For a rooted tree $T$, let $\Lambda(T)$ denote the number of leaves of $T$. For the case when $T$ contains only one node, let $\Lambda(T):=0$. With $\alpha$, we denote the inverse Ackermann function (see, e. g., [9]). It is well known that $\alpha$ grows extremely slowly. For $x=10^{80}$ (estimated number of atoms in the universe), $\alpha(x) \leq 4$.

\section{Using a Layering Partition}

The concept of a layering partition was introduced in [4]5]. The idea is the following. First, partition the vertices of a given graph $G=(V, E)$ in distance layers $L_{i}=\left\{v \mid d_{G}(s, v)=i\right\}$ for a given vertex $s$. Second, partition each layer $L_{i}$ 
into clusters in such a way that two vertices $u$ and $v$ are in the same cluster if and only if they are connected by a path only using vertices in the same or upper layers. That is, $u$ and $v$ are in the same cluster if and only if, for some $i$, $\{u, v\} \subseteq L_{i}$ and there is a path $P$ from $u$ to $v$ in $G$ such that, for all $j<i$, $P \cap L_{j}=\emptyset$. Note that each cluster $C$ is a set of vertices of $G$, i. e., $C \subseteq V$, and all clusters are pairwise disjoint. The created clusters form a rooted tree $\mathcal{T}$ with the cluster $\{s\}$ as the root where each cluster is a node of $\mathcal{T}$ and two clusters $C$ and $C^{\prime}$ are adjacent in $\mathcal{T}$ if and only if $G$ contains an edge $u v$ with $u \in C$ and $v \in C^{\prime}$. Figure 1 gives an example for such a partition. A layering partition of a graph can be computed in linear time [5].

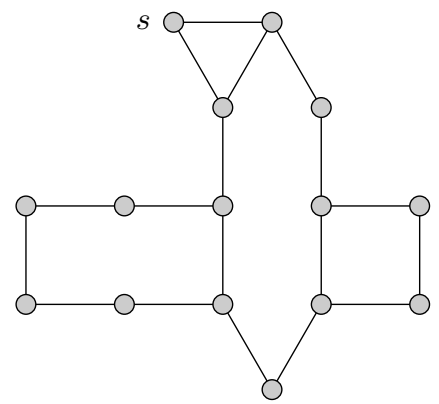

(a) A graph $G$.

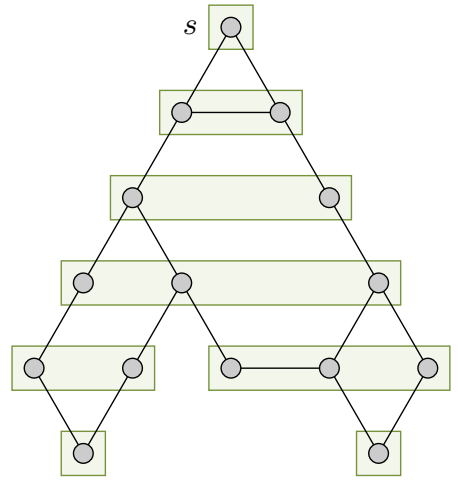

(b) A layering partition $\mathcal{T}$ for $G$.

Fig. 1. Example of a layering partition. A given graph $G($ a) and the layering partition of $G$ generated when starting at vertex $s$ (b). Example taken from [5].

For the remainder of this section, assume that we are given a graph $G=(V, E)$ and a layering partition $\mathcal{T}$ of $G$ for an arbitrary start vertex. We denote the largest diameter of all clusters of $\mathcal{T}$ as $\Delta$, i.e., $\Delta:=\max \left\{d_{G}(x, y) \mid\right.$ $x, y$ are in a cluster $C$ of $\mathcal{T}\}$. For two vertices $u$ and $v$ of $G$ contained in the clusters $C_{u}$ and $C_{v}$ of $\mathcal{T}$, respectively, we define $d_{\mathcal{T}}(u, v):=d_{\mathcal{T}}\left(C_{u}, C_{v}\right)$.

Lemma 1. For all vertices $u$ and $v$ of $G, d_{\mathcal{T}}(u, v) \leq d_{G}(u, v) \leq d_{\mathcal{T}}(u, v)+\Delta$.

Proof. Clearly, by construction of a layering partition, $d_{\mathcal{T}}(u, v) \leq d_{G}(u, v)$ for all vertices $u$ and $v$ of $G$.

Next, let $C_{u}$ and $C_{v}$ be the clusters containing $u$ and $v$, respectively. Note that $\mathcal{T}$ is a rooted tree. Let $C^{\prime}$ be the lowest common ancestor of $C_{u}$ and $C_{v}$. Therefore, $d_{\mathcal{T}}(u, v)=d_{\mathcal{T}}\left(u, C^{\prime}\right)+d_{\mathcal{T}}\left(C^{\prime}, v\right)$. By construction of a layering partition, $C^{\prime}$ contains a vertex $u^{\prime}$ and vertex $v^{\prime}$ such that $d_{G}\left(u, u^{\prime}\right)=d_{\mathcal{T}}\left(u, u^{\prime}\right)$ and $d_{G}\left(v, v^{\prime}\right)=$ $d_{\mathcal{T}}\left(v, v^{\prime}\right)$. Since the diameter of each cluster is at most $\Delta, d_{G}(u, v) \leq d_{\mathcal{T}}\left(u, u^{\prime}\right)+$ $\Delta+d_{\mathcal{T}}\left(v, v^{\prime}\right)=d_{\mathcal{T}}(u, v)+\Delta$. 
Theorem 1 below shows that we can use the layering partition $\mathcal{T}$ to compute an $(r+\Delta)$-dominating set for $G$ in linear time which is not larger than a minimum $r$-dominating set for $G$. This is done by finding a minimum $r$-dominating set of $\mathcal{T}$ where, for each cluster $C$ of $\mathcal{T}, r(C)$ is defined as $\min _{v \in C} r(v)$.

Theorem 1. Let $D$ be a minimum $r$-dominating set for a given graph $G$. An $(r+$ $\Delta$ )-dominating set $D^{\prime}$ for $G$ with $\left|D^{\prime}\right| \leq|D|$ can be computed in linear time.

Proof. First, create a layering partition $\mathcal{T}$ of $G$ and, for each cluster $C$ of $\mathcal{T}$, set $r(C):=\min _{v \in C} r(v)$. Second, find a minimum $r$-dominating set $\mathcal{S}$ for $\mathcal{T}$, i. e., a set $\mathcal{S}$ of clusters such that, for each cluster $C$ of $\mathcal{T}, d_{\mathcal{T}}(C, \mathcal{S}) \leq r(C)$. Third, create a set $D^{\prime}$ by picking an arbitrary vertex of $G$ from each cluster in $\mathcal{S}$. All three steps can be performed in linear time, including the computation of $\mathcal{S}$ (see [3]).

Next, we show that $D^{\prime}$ is an $(r+\Delta)$-dominating set for $G$. By construction of $\mathcal{S}$, each cluster $C$ of $\mathcal{T}$ has distance at most $r(C)$ to $\mathcal{S}$ in $\mathcal{T}$. Thus, for each vertex $u$ of $G, \mathcal{S}$ contains a cluster $C_{\mathcal{S}}$ with $d_{\mathcal{T}}\left(u, C_{\mathcal{S}}\right) \leq r(u)$. Additionally, by Lemma $11 d_{G}(u, v) \leq r(u)+\Delta$ for any vertex $v \in C_{\mathcal{S}}$. Therefore, for any vertex $u$, $d_{G}\left(u, D^{\prime}\right) \leq r(u)+\Delta$, i. e., $D^{\prime}$ is an $(r+\Delta)$-dominating set for $G$.

It remains to show that $\left|D^{\prime}\right| \leq|D|$. Let $\mathcal{D}$ be the set of clusters of $\mathcal{T}$ that contain a vertex of $D$. Because $D$ is an $r$-dominating set for $G$, it follows from Lemma 1 that $\mathcal{D}$ is an $r$-dominating set for $\mathcal{T}$. Clearly, since clusters are pairwise disjoint, $|\mathcal{D}| \leq|D|$. By minimality of $\mathcal{S},|\mathcal{S}| \leq|\mathcal{D}|$ and, by construction of $D^{\prime}$, $\left|D^{\prime}\right|=|\mathcal{S}|$. Therefore, $\left|D^{\prime}\right| \leq|D|$.

We now show how to construct a connected $(r+2 \Delta)$-dominating set for $G$ using $\mathcal{T}$ in such a way that the set created is not larger than a minimum connected $r$-dominating set for $G$. For the remainder of this section, let $D_{r}$ be a minimum connected $r$-dominating set of $G$ and let, for each cluster $C$ of $\mathcal{T}, r(C)$ be defined as above. Additionally, we say that a subtree $T^{\prime}$ of some tree $T$ is an $r$-dominating subtree of $T$ if the nodes (clusters in case of a layering partition) of $T^{\prime}$ form a connected $r$-dominating set for $T$.

The first step of our approach is to construct a minimum $r$-dominating subtree $T_{r}$ of $\mathcal{T}$. Such a subtree $T_{r}$ can be computed in linear time [12. Lemma 2 below shows that $T_{r}$ gives a lower bound for the cardinality of $D_{r}$.

Lemma 2. If $T_{r}$ contains more than one cluster, each connected $r$-dominating set of $G$ intersects all clusters of $T_{r}$. Therefore, $\left|T_{r}\right| \leq\left|D_{r}\right|$.

Proof. Let $D$ be an arbitrary connected $r$-dominating set of $G$. Assume that $T_{r}$ has a cluster $C$ such that $C \cap D=\emptyset$. Because $D$ is connected, the subtree of $\mathcal{T}$ induced by the clusters intersecting $D$ is connected, too. Thus, if $D$ intersects all leafs of $T_{r}$, then it intersects all clusters of $T_{r}$. Hence, we can assume, without loss of generality, that $C$ is a leaf of $T_{r}$. Because $T_{r}$ has at least two clusters and by minimality of $T_{r}$, $\mathcal{T}$ contains a cluster $C^{\prime}$ such that $d_{\mathcal{T}}\left(C^{\prime}, C\right)=d_{\mathcal{T}}\left(C^{\prime}, T_{r}\right)=r\left(C^{\prime}\right)$. Note that each path in $G$ from a vertex in $C^{\prime}$ to a vertex in $D$ intersects $C$. Therefore, by Lemma 1 there is a vertex $u \in C^{\prime}$ with $r(u)=d_{\mathcal{T}}(u, C)<d_{\mathcal{T}}(u, D) \leq d_{G}(u, D)$. That contradicts with $D$ being an $r$-dominating set. 
Because any $r$-dominating set of $G$ intersects each cluster of $T_{r}$ and because these clusters are pairwise disjoint, it follows that $\left|T_{r}\right| \leq\left|D_{r}\right|$.

As we show later in Corollary 1 each connected vertex set $S \subseteq V$ that intersects each cluster of $T_{r}$ gives an $(r+\Delta)$-dominating set for $G$. It follows from Lemma 2 that, if such a set $S$ has minimum cardinality, $|S| \leq\left|D_{r}\right|$. However, finding a minimum cardinality connected set intersecting each cluster of a layering partition (or of a subtree of it) is as hard as finding a minimum Steiner tree.

The main idea of our approach is to construct a minimum $(r+\delta)$-dominating subtree $T_{\delta}$ of $\mathcal{T}$ for some integer $\delta$. We then compute a small enough connected set $S_{\delta}$ that intersects all cluster of $T_{\delta}$. By trying different values of $\delta$, we eventually construct a connected set $S_{\delta}$ such that $\left|S_{\delta}\right| \leq\left|T_{r}\right|$ and, thus, $\left|S_{\delta}\right| \leq\left|D_{r}\right|$. Additionally, we show that $S_{\delta}$ is a connected $(r+2 \Delta)$-dominating set of $G$.

For some non-negative integer $\delta$, let $T_{\delta}$ be a minimum $(r+\delta)$-dominating subtree of $\mathcal{T}$. Clearly, $T_{0}=T_{r}$. The following two lemmas set an upper bound for the maximum distance of a vertex of $G$ to a vertex in a cluster of $T_{\delta}$ and for the size of $T_{\delta}$ compared to the size of $T_{r}$.

Lemma 3. For each vertex $v$ of $G, d_{\mathcal{T}}\left(v, T_{\delta}\right) \leq r(v)+\delta$.

Proof. Let $C_{v}$ be the cluster of $\mathcal{T}$ containing $v$ and let $C$ be the cluster of $T_{\delta}$ closest to $C_{v}$ in $\mathcal{T}$. By construction of $T_{\delta}, d_{\mathcal{T}}(v, C)=d_{\mathcal{T}}\left(C_{v}, C\right) \leq r\left(C_{v}\right)+\delta \leq$ $r(v)+\delta$.

Because the diameter of each cluster is at most $\Delta$, Lemma 1 and Lemma 3 imply the following.

Corollary 1. If a vertex set intersects all clusters of $T_{\delta}$, it is an $(r+(\delta+\Delta))$ dominating set of $G$.

Lemma 4. $\left|T_{\delta}\right| \leq\left|T_{r}\right|-\delta \cdot \Lambda\left(T_{\delta}\right)$.

Proof. First, consider the case when $T_{\delta}$ contains only one cluster, i. e., $\left|T_{\delta}\right|=1$. Then, $\Lambda\left(T_{\delta}\right)=1$ and, thus, the statement clearly holds. Next, let $T_{\delta}$ contain more than one cluster, let $C_{u}$ be an arbitrary leaf of $T_{\delta}$, and let $C_{v}$ be a cluster of $T_{r}$ with maximum distance to $C_{u}$ such that $C_{u}$ is the only cluster on the shortest path from $C_{u}$ to $C_{v}$ in $T_{r}$, i.e., $C_{v}$ is not in $T_{\delta}$. Due to the minimality of $T_{\delta}$, $d_{T_{r}}\left(C_{u}, C_{v}\right)=\delta$. Thus, the shortest path from $C_{u}$ to $C_{v}$ in $T_{r}$ contains $\delta$ clusters (including $C_{v}$ ) which are not in $T_{\delta}$. Therefore, $\left|T_{\delta}\right| \leq\left|T_{r}\right|-\delta \cdot \Lambda\left(T_{\delta}\right)$.

Now that we have constructed and analysed $T_{\delta}$, we show how to construct $S_{\delta}$. First, we construct a set of shortest paths such that each cluster of $T_{\delta}$ is intersected by exactly one path. Second, we connect these paths with each other to from a connected set using an approach which is similar to Kruskal's algorithm for minimum spanning trees.

Let $\mathcal{L}=\left\{C_{1}, C_{2}, \ldots, C_{\lambda}\right\}$ be the leaf clusters of $T_{\delta}$ (excluding the root) with either $\lambda=\Lambda\left(T_{\delta}\right)-1$ if the root of $T_{\delta}$ is a leaf, or with $\lambda=\Lambda\left(T_{\delta}\right)$ otherwise. We construct a set $\mathcal{P}=\left\{P_{1}, P_{2}, \ldots, P_{\lambda}\right\}$ of paths as follows. Initially, $\mathcal{P}$ is empty. 
For each cluster $C_{i} \in \mathcal{L}$, in turn, find the ancestor $C_{i}^{\prime}$ of $C_{i}$ which is closest to the root of $T_{\delta}$ and does not intersect any path in $\mathcal{P}$ yet. If we assume that the indices of the clusters in $\mathcal{L}$ represent the order in which they are processed, then $C_{1}^{\prime}$ is the root of $T_{\delta}$. Then, select an arbitrary vertex $v$ in $\mathcal{C}_{i}$ and find a shortest path $P_{i}$ in $G$ form $v$ to $C_{i}^{\prime}$. Add $P_{i}$ to $\mathcal{P}$ and continue with the next cluster in $\mathcal{L}$. Figure 2 gives an example.

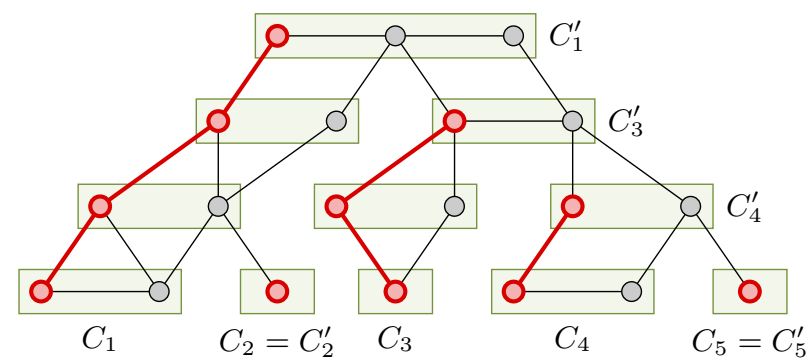

Fig. 2. Example for the set $\mathcal{P}$ for a subtree of a layering partition. Paths are shown in red. Each path $P_{i}$, with $1 \leq i \leq 5$, starts in the leaf $C_{i}$ and ends in the cluster $C_{i}^{\prime}$. For $i=2$ and $i=5, P_{i}$ contains only one vertex.

Lemma 5. For each cluster $C$ of $T_{\delta}$, there is exactly one path $P_{i} \in \mathcal{P}$ intersecting $C$. Additionally, $C$ and $P_{i}$ share exactly one vertex, i.e., $\left|C \cap P_{i}\right|=1$.

Proof. Observe that, by construction of a layering partition, each vertex in a cluster $C$ is adjacent to some vertex in the parent cluster of $C$. Therefore, a shortest path $P$ in $G$ from $C$ to any of its ancestors $C^{\prime}$ only intersects clusters on the path from $C$ to $C^{\prime}$ in $\mathcal{T}$ and each cluster shares only one vertex with $P$. It remains to show that each cluster intersects exactly one path.

Without loss of generality, assume that the indices of clusters in $\mathcal{L}$ and paths in $\mathcal{P}$ represent the order in which they are processed and created, i. e., assume that the algorithms first creates $P_{1}$ which starts in $C_{1}$, then $P_{2}$ which starts in $C_{2}$, and so on. Additionally, let $\mathcal{L}_{i}=\left\{C_{1}, C_{2}, \ldots, C_{i}\right\}$ and $\mathcal{P}_{i}=\left\{P_{1}, P_{2}, \ldots, P_{i}\right\}$.

To proof that each cluster intersects exactly one path, we show by induction over $i$ that, if a cluster $C_{i}$ of $T_{\delta}$ satisfies the statement, then all ancestors of $C_{i}$ satisfy it, too. Thus, if $C_{\lambda}$ satisfies the statement, each cluster satisfies it.

First, consider $i=1$. Clearly, since $P_{1}$ is the first path, $P_{1}$ connects the leaf $C_{1}$ with the root of $T_{\delta}$ and no cluster intersects more than one path at this point. Therefore, the statement is true for $C_{1}$ and each of its ancestors.

Next, assume that $i>1$ and that the statement is true for each cluster in $\mathcal{L}_{i-1}$ and their respective ancestors. Then, the algorithm creates $P_{i}$ which connects the leaf $C_{i}$ with the cluster $C_{i}^{\prime}$. Assume that there is a cluster $C$ on the path from $C_{i}$ to $C_{i}^{\prime}$ in $\mathcal{T}$ such that $C$ intersects a path $P_{j}$ with $j<i$. Clearly, $C_{i}^{\prime}$ is an ancestor of $C$. Thus, by induction hypothesis, $C_{i}^{\prime}$ is also intersected by some 
path $P \neq P_{i}$. This contradicts with the way $C_{i}^{\prime}$ is selected by the algorithm. Therefore, each cluster on the path from $C_{i}$ to $C_{i}^{\prime}$ in $\mathcal{T}$ only intersects $P_{i}$ and $P_{i}$ does not intersect any other clusters.

Because $i>1, C_{i}^{\prime}$ has a parent cluster $C^{\prime \prime}$ in $T_{\delta}$ that is intersected by a path $P_{j}$ with $j<i$. By induction hypothesis, each ancestor of $C^{\prime \prime}$ is intersected by a path in $\mathcal{P}_{i-1}$. Therefore, each ancestor of $C_{i}$ is intersected by exactly one path in $\mathcal{P}_{i}$.

Next, we use the paths in $\mathcal{P}$ to create the set $S_{\delta}$. As first step, let $S_{\delta}:=$ $\bigcup_{P_{i} \in \mathcal{P}} P_{i}$. Later, we add more vertices into $S_{\delta}$ to ensure it is a connected set.

Now, create a partition $\mathcal{V}=\left\{V_{1}, V_{2}, \ldots, V_{\lambda}\right\}$ of $V$ such that, for each $i$, $P_{i} \subseteq V_{i}, V_{i}$ is connected, and $d_{G}\left(v, P_{i}\right)=\min _{P \in \mathcal{P}} d_{G}(v, P)$ for each vertex $v \in V_{i}$. That is, $V_{i}$ contains the vertices of $G$ which are not more distant to $P_{i}$ in $G$ than to any other path in $\mathcal{P}$. Additionally, for each vertex $v \in V$, set $P(v):=P_{i}$ if and only if $v \in V_{i}$ (i.e., $P(v)$ is the path in $\mathcal{P}$ which is closest to $v$ ) and set $d(v):=d_{G}(v, P(v))$. Such a partition as well as $P(v)$ and $d(v)$ can be computed by performing a BFS on $G$ starting at all paths $P_{i} \in \mathcal{P}$ simultaneously. Later, the BFS also allows us to easily determine the shortest path from $v$ to $P(v)$ for each vertex $v$.

To manage the subsets of $\mathcal{V}$, we use a Union-Find data structure such that, for two vertices $u$ and $v, \operatorname{Find}(u)=\operatorname{Find}(v)$ if and only if $u$ and $v$ are in the same set of $\mathcal{V}$. A Union-Find data structure additionally allows us to easily join two set of $\mathcal{V}$ into one by performing a single Union operation. Note that, whenever we join two sets of $\mathcal{V}$ into one, $P(v)$ and $d(v)$ remain unchanged for each vertex $v$.

Next, create an edge set $E^{\prime}=\{u v \mid \operatorname{Find}(u) \neq \operatorname{Find}(v)\}$, i. e., the set of edges $u v$ such that $u$ and $v$ are in different sets of $\mathcal{V}$. Sort $E^{\prime}$ in such a way that an edge $u v$ precedes an edge $x y$ only if $d(u)+d(v) \leq d(x)+d(y)$.

The last step to create $S_{\delta}$ is similar to Kruskal's minimum spanning tree algorithm. Iterate over the edges in $E^{\prime}$ in increasing order. If, for an edge $u v$, Find $(u) \neq \operatorname{Find}(v)$, i. e., if $u$ and $v$ are in different sets of $\mathcal{V}$, then join these sets into one by performing $\operatorname{Union}(u, v)$, add the vertices on the shortest path from $u$ to $P(u)$ to $S_{\delta}$, and add the vertices on the shortest path from $v$ to $P(v)$ to $S_{\delta}$. Repeat this, until $\mathcal{V}$ contains only one set, i. e., until $\mathcal{V}=\{V\}$.

Algorithm 1 below summarises the steps to create a set $S_{\delta}$ for a given subtree of a layering partition subtree $T_{\delta}$.

Lemma 6. For a given graph $G$ and a given subtree $T_{\delta}$ of some layering partition of $G$, Algorithm 1 constructs, in $\mathcal{O}(m \alpha(n))$ time, a connected set $S_{\delta}$ with $\left|S_{\delta}\right| \leq$ $\left|T_{\delta}\right|+\Delta \cdot \Lambda\left(T_{\delta}\right)$ which intersects each cluster of $T_{\delta}$.

Proof (Correctness). First, we show that $S_{\delta}$ is connected at the end of the algorithm. To do so, we show by induction that, at any time, $S_{\delta} \cap V^{\prime}$ is a connected set for each set $V^{\prime} \in \mathcal{V}$. Clearly, when $\mathcal{V}$ is created, for each set $V_{i} \in \mathcal{V}$, $S_{\delta} \cap V_{i}=P_{i}$. Now, assume that the algorithm joins the set $V_{u}$ and $V_{v}$ in $\mathcal{V}$ into one set based on the edge $u v$ with $u \in V_{u}$ and $v \in V_{v}$. Let $S_{u}=S_{\delta} \cap V_{u}$ and $S_{v}=S_{\delta} \cap V_{v}$. Note that $P(u) \subseteq S_{u}$ and $P(v) \subseteq S_{v}$. The algorithm now adds all 


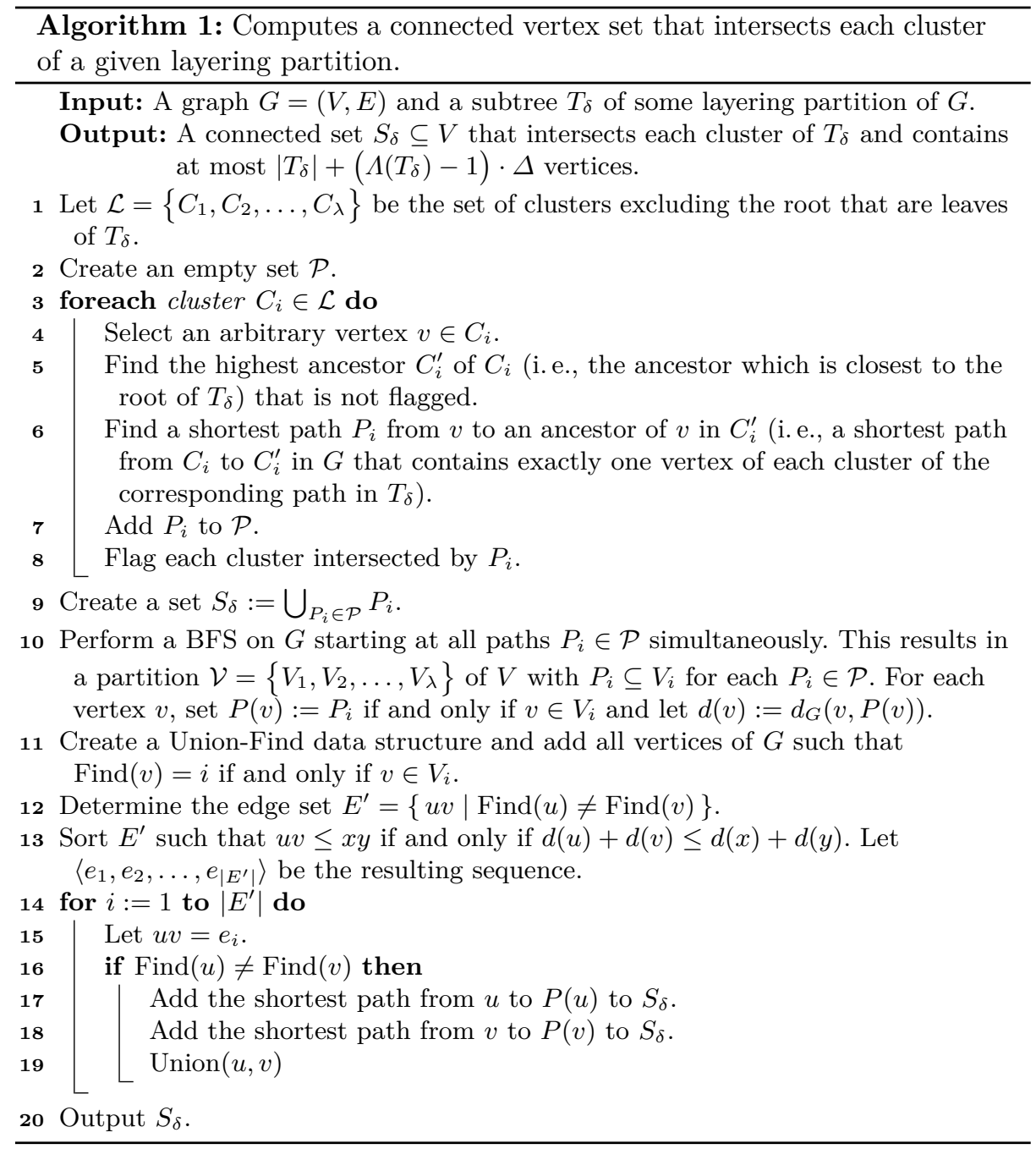

vertices to $S_{\delta}$ which are on a path from $P(u)$ to $P(v)$. Therefore, $S_{\delta} \cap\left(V_{u} \cup V_{v}\right)$ is a connected set. Because $\mathcal{V}=\{V\}$ at the end of the algorithm, $S_{\delta}$ is connected eventually. Additionally, since $P_{i} \subseteq S_{\delta}$ for each $P_{i} \in \mathcal{P}$, it follows that $S_{\delta}$ intersects each cluster of $T_{\delta}$.

Next, we show that the cardinality of $S_{\delta}$ is at most $\left|T_{\delta}\right|+\Delta \cdot \Lambda\left(T_{\delta}\right)$. When first created, the set $S_{\delta}$ contains all vertices of all paths in $\mathcal{P}$. Therefore, by Lemma 5. $\left|S_{\delta}\right|=\sum_{P_{i} \in \mathcal{P}}\left|P_{i}\right|=\left|T_{\delta}\right|$. Then, each time two sets of $\mathcal{V}$ are joined into one set based on an edge $u v, S_{\delta}$ is extended by the vertices on the shortest paths from $u$ to $P(u)$ and from $v$ to $P(v)$. Therefore, the size of $S_{\delta}$ increases by $d(u)+d(v)$, i. e., $\left|S_{\delta}\right|:=\left|S_{\delta}\right|+d(u)+d(v)$. Let $X$ denote the set of all edges used to join two sets of $\mathcal{V}$ into one at some point during the algorithm. Note that 
$|X|=|\mathcal{P}|-1 \leq \Lambda\left(T_{\delta}\right)$. Therefore, at the end of the algorithm,

$$
\left|S_{\delta}\right|=\sum_{P_{i} \in \mathcal{P}}\left|P_{i}\right|+\sum_{u v \in X}(d(u)+d(v)) \leq\left|T_{\delta}\right|+\Lambda\left(T_{\delta}\right) \cdot \max _{u v \in X}(d(u)+d(v)) .
$$

Claim. For each edge $u v \in X, d(u)+d(v) \leq \Delta$.

Proof (Claim). To represent the relations between paths in $\mathcal{P}$ and vertex sets in $\mathcal{V}$, we define a function $f: \mathcal{P} \rightarrow \mathcal{V}$ such that $f\left(P_{i}\right)=V_{j}$ if and only if $P_{i} \subseteq V_{j}$. Directly after constructing $\mathcal{V}, f$ is a bijection with $f\left(P_{i}\right)=V_{i}$. At the end of the algorithm, after all sets of $\mathcal{V}$ are joined into one, $f\left(P_{i}\right)=V$ for all $P_{i} \in \mathcal{P}$.

Recall the construction of $\mathcal{P}$ and assume that the indices of the paths in $\mathcal{P}$ represent the order in which they are created. Assume that $i>1$. By construction, the path $P_{i} \in \mathcal{P}$ connects the leaf $C_{i}$ with the cluster $C_{i}^{\prime}$ in $T_{\delta}$. Because $i>1$, $C_{i}^{\prime}$ has a parent cluster in $T_{\delta}$ that is intersected by a path $P_{j} \in \mathcal{P}$ with $j<i$. We define $P_{j}$ as the parent of $P_{i}$. By Lemma 5 this parent $P_{j}$ is unique for each $P_{i} \in \mathcal{P}$ with $i>1$. Based on this relation between paths in $\mathcal{P}$, we can construct a rooted tree $\mathbb{T}$ with the node set $\left\{x_{i} \mid P_{i} \in \mathcal{P}\right\}$ such that each node $x_{i}$ represents the path $P_{i}$ and $x_{j}$ is the parent of $x_{i}$ if and only if $P_{j}$ is the parent of $P_{i}$.

Because each node of $\mathbb{T}$ represents a path in $\mathcal{P}, f$ defines a colouring for the nodes of $\mathbb{T}$ such that $x_{i}$ and $x_{j}$ have different colours if and only if $f\left(P_{i}\right) \neq f\left(P_{j}\right)$. As long as $|\mathcal{V}|>1, \mathbb{T}$ contains two adjacent nodes with different colours. Let $x_{i}$ and $x_{j}$ be these nodes with $j<i$ and let $P_{i}$ and $P_{j}$ be the corresponding paths in $\mathcal{P}$. Note that $x_{j}$ is the parent of $x_{i}$ in $\mathbb{T}$ and, hence, $P_{j}$ is the parent of $P_{i}$. Therefore, $P_{i}$ ends in a cluster $C_{i}^{\prime}$ which has a parent cluster $C$ that intersects $P_{j}$. By properties of layering partitions, it follows that $d_{G}\left(P_{i}, P_{j}\right) \leq \Delta+1$. Recall that, by construction, $d(v)=\min _{P \in \mathcal{P}} d_{G}(v, P)$ for each vertex $v$. Thus, for each edge $u v$ on a shortest path from $P_{i}$ to $P_{j}$ in $G$ (with $u$ being closer to $P_{i}$ than to $\left.P_{j}\right), d(u)+d(v) \leq d_{G}\left(u, P_{i}\right)+d_{G}\left(v, P_{j}\right) \leq \Delta$. Therefore, because $f\left(P_{i}\right) \neq f\left(P_{j}\right)$, there is an edge $u v$ on a shortest path from $P_{i}$ to $P_{j}$ such that $f(P(u)) \neq f(P(v))$ and $d(u)+d(v) \leq \Delta$.

From the claim above, it follows that, as long as $\mathcal{V}$ contains multiple sets, there is an edge $u v \in E^{\prime}$ such that $d(u)+d(v) \leq \Delta$ and Find $(u) \neq \operatorname{Find}(v)$. Therefore, $\max _{u v \in X}(d(u)+d(v)) \leq \Delta$ and $\left|S_{\delta}\right| \leq\left|T_{\delta}\right|+\left(\Lambda\left(T_{\delta}\right)-1\right) \cdot \Delta$.

Proof (Complexity). First, the algorithm computes $\mathcal{P}$ (line 2 to line 8). If the parent of each vertex from the original BFS that was used to construct $\mathcal{T}$ is still known, $\mathcal{P}$ can be constructed in $\mathcal{O}(n)$ total time. After picking a vertex $v$ in $C_{i}$, simply follow the parent pointers until a vertex in $C_{i}^{\prime}$ is reached. Computing $\mathcal{V}$ as well as $P(v)$ and $d(v)$ for each vertex $v$ of $G$ (line 10 can be done with single BFS and, thus, requires at most $\mathcal{O}(n+m)$ time.

Recall that, for a Union-Find data structure storing $n$ elements, each operation requires at most $\mathcal{O}(\alpha(n))$ amortised time. Therefore, initialising such a data structure to store all vertices (line 11) and computing $E^{\prime}$ (line 12) requires at most $\mathcal{O}(m \alpha(n))$ time. Note that, for each vertex $v, d(v) \leq|V|$. Thus, sorting $E^{\prime}$ (line 13 can be done in linear time using counting sort. When iterating over $E^{\prime}$ 
(line 14 to line 19p, for each edge $u v \in E^{\prime}$, the Find-operation is called twice and the Union-operation is called at most once. Thus, the total runtime for all these operations is at most $\mathcal{O}(m \alpha(n))$.

Let $P_{u}=\{u, \ldots, x, y, \ldots, p\}$ be the shortest path in $G$ from a vertex $u$ to $P(u)$. Assume that $y$ has been added to $S_{\delta}$ in a previous iteration. Thus, $\{y, \ldots, p\} \subseteq S_{\delta}$ and, when adding $P_{u}$ to $S_{\delta}$, the algorithm only needs to add $\{u, \ldots, x\}$. Therefore, by using a simple binary flag to determine if a vertex is contained in $S_{\delta}$, constructing $S_{\delta}$ (line 9 line 17 and line 18) requires at most $\mathcal{O}(n)$ time.

In total, Algorithm 1 runs in $\mathcal{O}(m \alpha(n))$ time.

Because, for each integer $\delta \geq 0,\left|S_{\delta}\right| \leq\left|T_{\delta}\right|+\Delta \cdot \Lambda\left(T_{\delta}\right)$ (Lemma 6) and $\left|T_{\delta}\right| \leq\left|T_{r}\right|-\delta \cdot \Lambda\left(T_{\delta}\right)$ (Lemma 4 , we have the following.

Corollary 2. For each $\delta \geq \Delta,\left|S_{\delta}\right| \leq\left|T_{r}\right|$ and, thus, $\left|S_{\delta}\right| \leq\left|D_{r}\right|$.

To the best of our knowledge, there is no algorithm known that computes $\Delta$ in less than $\mathcal{O}(\mathrm{nm})$ time. Additionally, under reasonable assumptions, computing the diameter or radius of a general graph requires $\Omega\left(n^{2}\right)$ time [1]. We conjecture that the runtime for computing $\Delta$ for a given graph has a similar lower bound.

To avoid the runtime required for computing $\Delta$, we use the following approach shown in Algorithm 2 below. First, compute a layering partition $\mathcal{T}$ and the subtree $T_{r}$. Second, for a certain value of $\delta$, compute $T_{\delta}$ and perform Algorithm 1 on it. If the resulting set $S_{\delta}$ is larger than $T_{r}$ (i.e., $\left|S_{\delta}\right|>\left|T_{r}\right|$ ), increase $\delta$; otherwise, if $\left|S_{\delta}\right| \leq\left|T_{r}\right|$, decrease $\delta$. Repeat the second step with the new value of $\delta$.

One strategy to select values for $\delta$ is a classical binary search over the number of vertices of $G$. In this case, Algorithm 1 is called up-to $\mathcal{O}(\log n)$ times. Empirical analysis [2, however, have shown that $\Delta$ is usually very small. Therefore, we use a so-called one-sided binary search.

Consider a sorted sequence $\left\langle x_{1}, x_{2}, \ldots, x_{n}\right\rangle$ in which we search for a value $x_{p}$. We say the value $x_{i}$ is at position $i$. For a one-sided binary search, instead of starting in the middle at position $n / 2$, we start at position 1 . We then processes position 2 , then position 4 , then position 8 , and so on until we reach position $j=2^{i}$ and, next, position $k=2^{i+1}$ with $x_{j}<x_{p} \leq x_{k}$. Then, we perform a classical binary search on the sequence $\left\langle x_{j+1}, \ldots, x_{k}\right\rangle$. Note that, because $x_{j}<x_{p} \leq x_{k}$, $2^{i}<p \leq 2^{i+1}$ and, hence, $j<p \leq k<2 p$. Therefore, a one-sided binary search requires at most $\mathcal{O}(\log p)$ iterations to find $x_{p}$.

Because of Corollary 2, using a one-sided binary search allows us to find a value $\delta \leq \Delta$ for which $\left|S_{\delta}\right| \leq\left|T_{r}\right|$ by calling Algorithm 1 at most $\mathcal{O}(\log \Delta)$ times. Algorithm 2 below implements this approach.

Theorem 2. For a given graph $G$, Algorithm 2 computes a connected $(r+2 \Delta)$ dominating set $D$ with $|D| \leq\left|D_{r}\right|$ in $\mathcal{O}(m \alpha(n) \log \Delta)$ time.

Proof. Clearly, the set $D$ is connected because $D=S_{\delta}$ for some $\delta$ and, by Lemma 6 the set $S_{\delta}$ is connected. By Corollary 2 for each $\delta \geq \Delta,\left|S_{\delta}\right| \leq\left|T_{r}\right|$. 


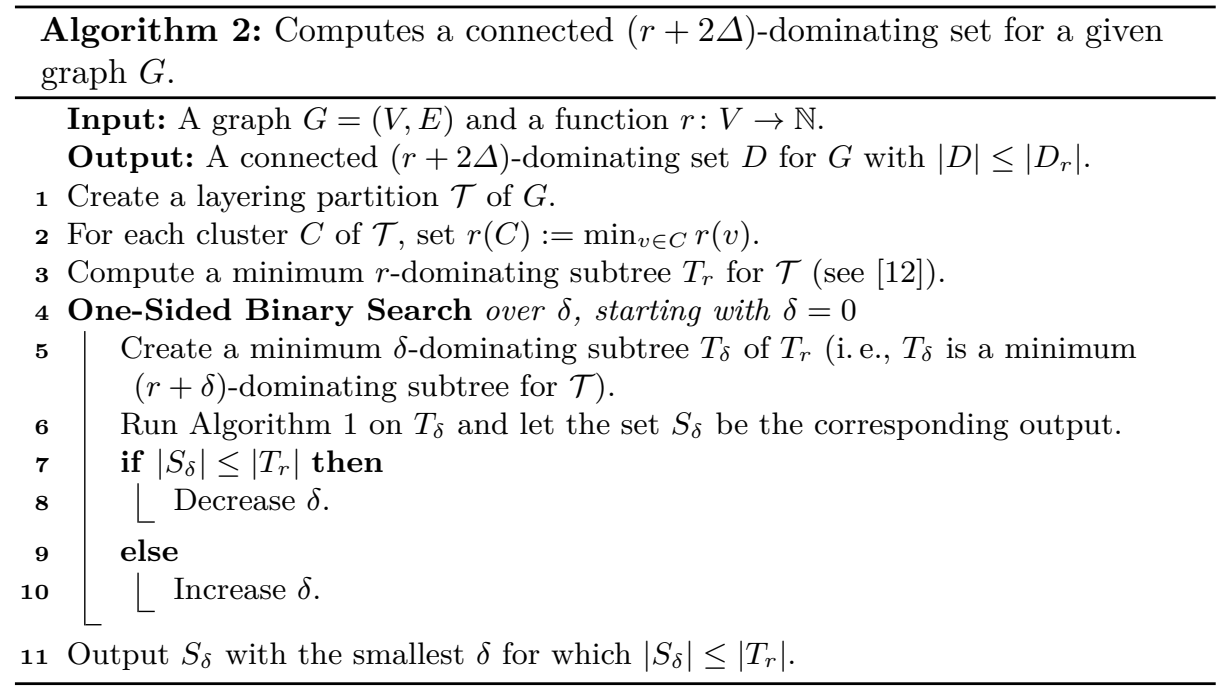

Thus, for each $\delta \geq \Delta$, the binary search decreases $\delta$ and, eventually, finds some $\delta$ such that $\delta \leq \Delta$ and $\left|S_{\delta}\right| \leq\left|T_{r}\right|$. Therefore, the algorithm finds a set $D$ with $|D| \leq\left|D_{r}\right|$. Note that, because $D=S_{\delta}$ for some $\delta \leq \Delta$ and because $S_{\delta}$ intersects each cluster of $T_{\delta}$ (Lemma 6 ), it follows from Lemma 3 that, for each vertex $v$ of $G, d_{\mathcal{T}}(v, D) \leq r(v)+\Delta$ and, by Lemma $1 . d_{G}(v, D) \leq r(v)+2 \Delta$. Thus, $D$ is an $(r+2 \Delta)$-dominating set for $G$.

Creating a layering partition for a given graph and computing a minimum connected $r$-dominating set of a tree can be done in linear time [12]. The onesided binary search over $\delta$ has at most $\mathcal{O}(\log \Delta)$ iterations. Each iteration of the binary search requires at most linear time to compute $T_{\delta}, \mathcal{O}(m \alpha(n))$ time to compute $S_{\delta}$ (Lemma 6), and constant time to decide whether to increase or decrease $\delta$. Therefore, Algorithm 2 runs in $\mathcal{O}(m \alpha(n) \log \Delta)$ total time.

\section{Using a Tree-Decomposition}

Theorem 1 and Theorem 2 respectively show how to compute an $(r+\Delta)$ dominating set in linear time and a connected $(r+2 \Delta)$-dominating set in $\mathcal{O}(m \alpha(n) \log \Delta)$ time. It is known that the maximum diameter $\Delta$ of clusters of any layering partition of a graph approximates the tree-breadth and tree-length of this graph. Indeed, for a graph $G$ with $\operatorname{tl}(G)=\lambda, \Delta \leq 3 \lambda[10$.

Corollary 3. Let $D$ be a minimum $r$-dominating set for a given graph $G$ with $\operatorname{tl}(G)=\lambda$. An $(r+3 \lambda)$-dominating set $D^{\prime}$ for $G$ with $\left|D^{\prime}\right| \leq|D|$ can be computed in linear time.

Corollary 4. Let $D$ be a minimum connected $r$-dominating set for a given graph $G$ with $\operatorname{tl}(G)=\lambda$. A connected $(r+6 \lambda)$-dominating set $D^{\prime}$ for $G$ with $\left|D^{\prime}\right| \leq$ $|D|$ can be computed in $\mathcal{O}(m \alpha(n) \log \lambda)$ time. 
In this section, we consider the case when we are given a tree-decomposition with breadth $\rho$ and length $\lambda$. We present algorithms to compute an $(r+\rho)$ dominating set as well as a connected $(r+\min (3 \lambda, 5 \rho))$-dominating set in $\mathcal{O}(n m)$ time.

For the remainder of this section, assume that we are given a graph $G=(V, E)$ and a tree-decomposition $\mathcal{T}$ of $G$ with breadth $\rho$ and length $\lambda$. We assume that $\rho$ and $\lambda$ are known and that, for each bag $B$ of $\mathcal{T}$, we know a vertex $c(B)$ with $B \subseteq N_{G}^{\rho}[c(B)]$. Let $\mathcal{T}$ be minimal, i. e., $B \nsubseteq B^{\prime}$ for any two bags $B$ and $B^{\prime}$. Thus, the number of bags is not exceeding the number vertices of $G$. Additionally, let each vertex of $G$ store a list of bags containing it and let each bag of $T$ store a list of vertices it contains. One can see this as a bipartite graph where one subset of vertices are the vertices of $G$ and the other subset are the bags of $\mathcal{T}$. Therefore, the total input size is in $\mathcal{O}(n+m+M)$ where $M \leq n^{2}$ is the sum of the cardinality of all bags of $\mathcal{T}$.

\subsection{Preprocessing}

Before approaching the (Connected) $r$-Domination problem, we compute a subtree $\mathcal{T}^{\prime}$ of $\mathcal{T}$ such that, for each vertex $v$ of $G, \mathcal{T}^{\prime}$ contains a bag $B$ with $d_{G}(v, B) \leq r(v)$. We call such a (not necessarily minimal) subtree an $r$-covering subtree of $\mathcal{T}$.

Let $T_{r}$ be a minimum $r$-covering subtree of $\mathcal{T}$. We do not know how to compute $T_{r}$ directly. However, if we are given a bag $B$ of $\mathcal{T}$, we can compute the smallest $r$-covering subtree $T_{B}$ which contains $B$. Then, we can identify a bag $B^{\prime}$ in $T_{B}$ for which we know it is a bag of $T_{r}$. Thus, we can compute $T_{r}$ by computing the smallest $r$-covering subtree which contains $B^{\prime}$.

The idea for computing $T_{B}$ is to determine, for each vertex $v$ of $G$, the bag $B_{v}$ of $\mathcal{T}$ for which $d_{G}\left(v, B_{v}\right) \leq r(v)$ and which is closet to $B$. Then, let $T_{B}$ be the smallest tree that contains all these bags $B_{v}$. Algorithm 3 below implements this approach.

Additionally to computing the tree $T_{B}$, we make it a rooted tree with $B$ as the root, give each vertex $v$ a pointer $\beta(v)$ to a bag of $T_{B}$, and give each bag $B^{\prime}$ a counter $\sigma\left(B^{\prime}\right)$. The pointer $\beta(v)$ identifies the bag $B_{v}$ which is closest to $B$ in $T_{B}$ and intersects the $r$-neighbourhood of $v$. The counter $\sigma\left(B^{\prime}\right)$ states the number of vertices $v$ with $\beta(v)=B^{\prime}$. Even though setting $\beta$ and $\sigma$ as well as rooting the tree are not necessary for computing $T_{B}$, we use it when computing an $(r+\rho)$-dominating set later.

Lemma 7. For a given tree-decomposition $\mathcal{T}$ and a given bag $B$ of $\mathcal{T}, A l$ gorithm 3 computes an r-covering subtree $T_{B}$ in $\mathcal{O}(\mathrm{nm})$ time such that $T_{B}$ contains $B$ and has a minimal number of bags.

Proof (Correctness). Note that, by construction of the set $\mathcal{B}$ (line 5 to line 7), $\mathcal{B}$ contains a bag $B_{u}$ for each vertex $u$ of $G$ such that $d_{G}\left(u, B_{u}\right) \leq r(u)$. Thus, each subtree of $\mathcal{T}$ which contains all bags of $\mathcal{B}$ is an $r$-covering subtree. To show the correctness of the algorithm, it remains to show that the smallest $r$-covering 


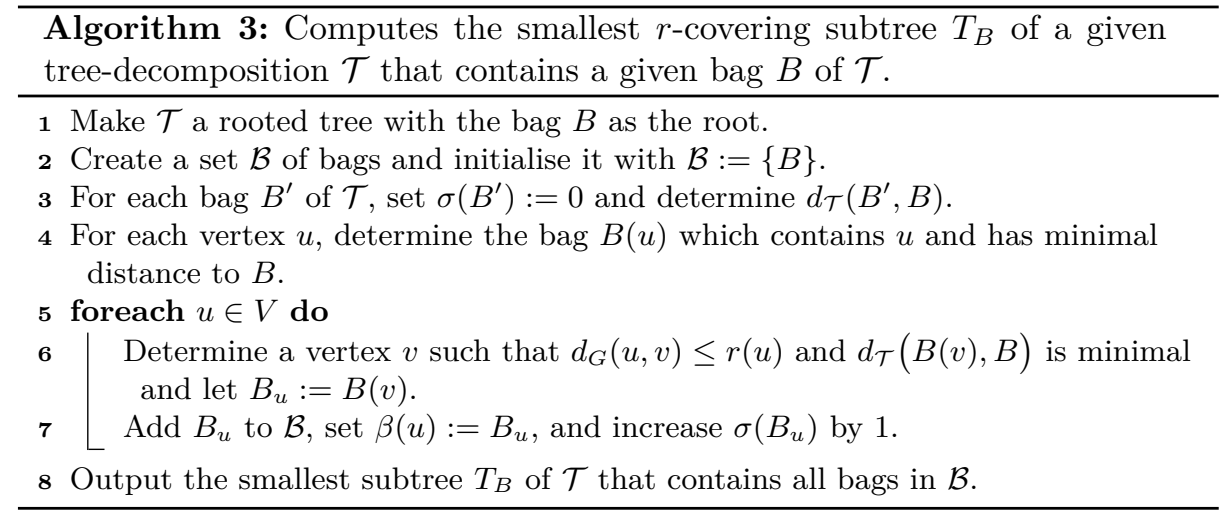

subtree of $\mathcal{T}$ which contains $B$ has to contain each bag from the set $\mathcal{B}$. Then, the subtree $T_{B}$ constructed in line 8 is the desired subtree.

By properties of tree-decompositions, the set of bags which intersect the $r$-neighbourhood of some vertex $u$ induces a subtree $T_{u}$ of $\mathcal{T}$. That is, $T_{u}$ contains exactly the bags $B^{\prime}$ with $d_{G}\left(u, B^{\prime}\right) \leq r(u)$. Note that $\mathcal{T}$ is a rooted tree with $B$ as the root. Clearly, the bag $B_{u} \in \mathcal{B}$ (determined in line 6) is the root of $T_{u}$ since it is the bag closest to $B$. Hence, each bag $B^{\prime}$ with $d_{G}\left(u, B^{\prime}\right) \leq r(u)$ is a descendant of $B_{u}$. Therefore, if a subtree of $\mathcal{T}$ contains $B$ and does not contain $B_{u}$, then it also cannot contain any descendant of $B_{u}$ and, thus, contains no bag intersecting the $r$-neighbourhood of $u$.

Proof (Complexity). Recall that $\mathcal{T}$ has at most $n$ bags and that the sum of the cardinality of all bags of $\mathcal{T}$ is $M \leq n^{2}$. Thus, line 3 and line 4 require at most $\mathcal{O}(M)$ time. Using a BFS, it takes at most $\mathcal{O}(m)$ time, for a given vertex $u$, to determine a vertex $v$ such that $d_{G}(u, v) \leq r(u)$ and $d_{\mathcal{T}}(B(v), B)$ is minimal (line 66. Therefore, the loop starting in line 5 and, thus, Algorithm 3 run in at most $\mathcal{O}(n m)$ total time.

Lemma 8 and Lemma 9 below show that each leaf $B^{\prime} \neq B$ of $T_{B}$ is a bag of a minimum $r$-covering subtree $T_{r}$ of $\mathcal{T}$. Note that both lemmas only apply if $T_{B}$ has at least two bags. If $T_{B}$ contains only one bag, it is clearly a minimum $r$-covering subtree.

Lemma 8. For each leaf $B^{\prime} \neq B$ of $T_{B}$, there is a vertex $v$ in $G$ such that $B^{\prime}$ is the only bag of $T_{B}$ with $d_{G}\left(v, B^{\prime}\right) \leq r(v)$.

Proof. Assume that Lemma 8 is false. Then, there is a leaf $B^{\prime}$ such that, for each vertex $v$ with $d_{G}\left(v, B^{\prime}\right) \leq r(v), T_{B}$ contains a bag $B^{\prime \prime} \neq B^{\prime}$ with $d_{G}\left(v, B^{\prime \prime}\right) \leq r(v)$. Thus, for each vertex $v$, the $r$-neighbourhood of $v$ is intersected by a bag of the tree-decomposition $T_{B}-B^{\prime}$. This contradicts with the minimality of $T_{B}$.

Lemma 9. For each leaf $B^{\prime} \neq B$ of $T_{B}$, there is a minimum $r$-covering subtree $T_{r}$ of $\mathcal{T}$ which contains $B^{\prime}$. 
Proof. Assume that $T_{r}$ is a minimum $r$-covering subtree which does not contain $B^{\prime}$. Because of Lemma 8 there is a vertex $v$ of $G$ such that $B^{\prime}$ is the only bag of $T_{B}$ which intersects the $r$-neighbourhood of $v$. Therefore, $T_{r}$ contains only bags which are descendants of $B^{\prime}$. Partition the vertices of $G$ into the sets $V^{\uparrow}$ and $V^{\downarrow}$ such that $V^{\downarrow}$ contains the vertices of $G$ which are contained in $B^{\prime}$ or in a descendant of $B^{\prime}$. Because $T_{r}$ is an $r$-covering subtree and because $T_{r}$ only contains descendants of $B^{\prime}$, it follows from properties of tree-decompositions that, for each vertex $v \in V^{\uparrow}$, there is a path of length at most $r(v)$ from $v$ to a bag of $T_{r}$ passing through $B^{\prime}$ and, thus, $d_{G}\left(v, B^{\prime}\right) \leq r(v)$. Similarly, since $T_{B}$ is an $r$-covering subtree, it follows that, for each vertex $v \in V^{\downarrow}, d_{G}\left(v, B^{\prime}\right) \leq r(v)$. Therefore, for each vertex $v$ of $G, d_{G}\left(v, B^{\prime}\right) \leq r(v)$ and, thus, $B^{\prime}$ induces an $r$-covering subtree $T_{r}$ of $\mathcal{T}$ with $\left|T_{r}\right|=1$.

Algorithm 4 below uses Lemma 9 to compute a minimum $r$-covering subtree $T_{r}$ of $\mathcal{T}$.

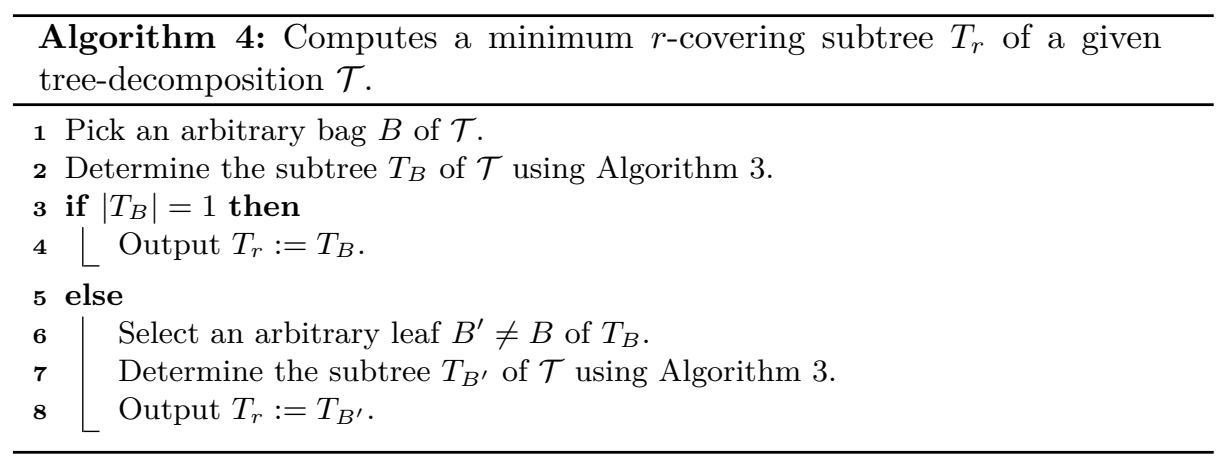

Lemma 10. Algorithm 4 computes a minimum $r$-covering subtree $T_{r}$ of $\mathcal{T}$ in $\mathcal{O}(n m)$ time.

Proof. Algorithm 4 first picks an arbitrary bag $B$ and then uses Algorithm 3 to compute the smallest $r$-covering subtree $T_{B}$ of $\mathcal{T}$ which contains $B$. By Lemma 9 . for each leaf $B^{\prime}$ of $T_{B}$, there is a minimum $r$-covering subtree $T_{r}$ which contains $B^{\prime}$. Thus, performing Algorithm 3 again with $B^{\prime}$ as input creates such a subtree $T_{r}$.

Clearly, with exception of calling Algorithm 3 , all steps of Algorithm 4 require only constant time. Because Algorithm 3 requires at most $\mathcal{O}(\mathrm{nm})$ time (see Lemma 7) and is called at most two times, Algorithm 4 runs in at most $\mathcal{O}(n m)$ total time.

Algorithm 4 computes $T_{r}$ by, first, computing $T_{B}$ for some bag $B$ and, second, computing $T_{B^{\prime}}=T_{r}$ for some leaf $B^{\prime}$ of $T_{B}$. Note that, because both trees are computed using Algorithm 3, Lemma 8 applies to $T_{B}$ and $T_{B^{\prime}}$. Therefore, we can slightly generalise Lemma 8 as follows. 
Corollary 5. For each leaf $B$ of $T_{r}$, there is a vertex $v$ in $G$ such that $B$ is the only bag of $T_{r}$ with $d_{G}(v, B) \leq r(v)$.

\section{$4.2 \quad r$-Domination}

In this subsection, we use the minimum $r$-covering subtree $T_{r}$ to determine an $(r+\rho)$-dominating set $S$ in $\mathcal{O}(n m)$ time using the following approach. First, compute $T_{r}$. Second, pick a leaf $B$ of $T_{r}$. If there is a vertex $v$ such that $v$ is not dominated and $B$ is the only bag intersecting the $r$-neighbourhood of $v$, then add the center of $B$ into $S$, flag all vertices $u$ with $d_{G}(u, B) \leq r(u)$ as dominated, and remove $B$ from $T_{r}$. Repeat the second step until $T_{r}$ contains no more bags and each vertex is flagged as dominated. Algorithm 5 below implements this approach. Note that, instead of removing bags from $T_{r}$, we use a reversed BFS-order of the bags to ensure the algorithm processes bags in the correct order.

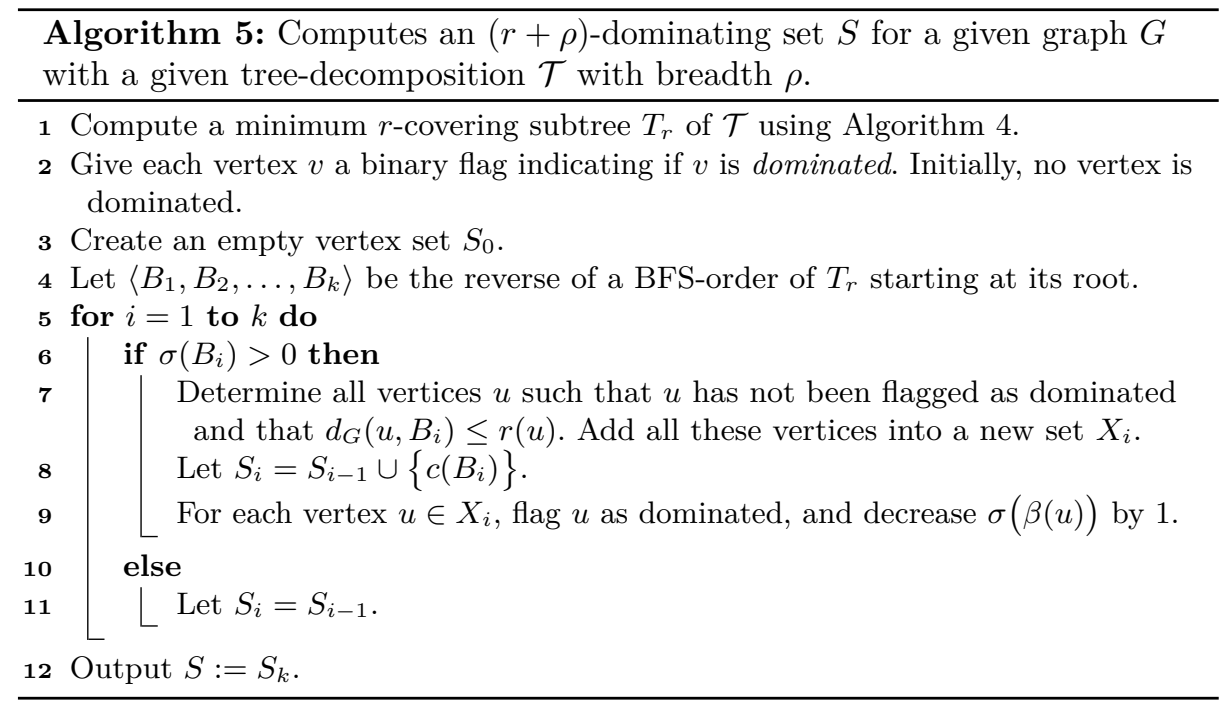

Theorem 3. Let $D$ be a minimum r-dominating set for a given graph $G$. Given a tree-decomposition with breadth $\rho$ for $G$, Algorithm 5 computes an $(r+\rho)$ dominating set $S$ with $|S| \leq|D|$ in $\mathcal{O}(n m)$ time.

Proof (Correctness). First, we show that $S$ is an $(r+\rho)$-dominating set for $G$. Note that a vertex $v$ is flagged as dominated only if $S_{i}$ contains a vertex $c\left(B_{j}\right)$ with $d_{G}\left(v, B_{j}\right) \leq r(v)$ (see line 7 to line 9 . Thus, $v$ is flagged as dominated only if $d_{G}\left(v, S_{i}\right) \leq d_{G}\left(v, c\left(B_{j}\right)\right) \leq r(v)+\rho$. Additionally, by construction of $T_{r}$ (see Algorithm 3), for each vertex $v, T_{r}$ contains a bag $B$ with $\beta(v)=B, \sigma(B)$ states the number of vertices $v$ with $\beta(v)=B$, and $\sigma(B)$ is decreased by 1 only if such 
a vertex $v$ is flagged as dominated (see line 9 ). Therefore, if $G$ contains a vertex $v$ with $d_{G}\left(v, S_{i}\right)>r(v)+\rho$, then $v$ is not flagged as dominated and $T_{r}$ contains a bag $B_{i}$ with $\beta(v)=B_{i}$ and $\sigma\left(B_{i}\right)>0$. Thus, when $B_{i}$ is processed by the algorithm, $c\left(B_{i}\right)$ will be added to $S_{i}$ and, hence, $d_{G}\left(v, S_{i}\right) \leq r(v)+\rho$.

Let $V_{i}^{S}=\left\{u \mid d_{G}\left(u, B_{j}\right) \leq r(u), c\left(B_{j}\right) \in S_{i}\right\}$ be the set of vertices which are flagged as dominated after the algorithm processed $B_{i}$, i. e., each vertex in $V_{i}^{S}$ is $(r+\rho)$-dominated by $S_{i}$. Similarly, for some set $D_{i} \subseteq D$, let $V_{i}^{D}=$ $\left\{u \mid d_{G}\left(u, D_{i}\right) \leq r(u)\right\}$ be the set of vertices dominated by $D_{i}$. To show that $|S| \leq|D|$, we show by induction over $i$ that, for each $i$, (i) there is a set $D_{i} \subseteq D$ such that $V_{i}^{D} \subseteq V_{i}^{S}$, (ii) $\left|S_{i}\right|=\left|D_{i}\right|$, and (iii) if, for some vertex $v, \beta(v)=B_{j}$ with $j \leq i$, then $v \in V_{i}^{S}$.

For the base case, let $S_{0}=D_{0}=\emptyset$. Then, $V_{0}^{S}=V_{0}^{D}=\emptyset$ and all three statements are satisfied. For the inductive step, first, consider the case when $\sigma\left(B_{i}\right)=0$. Because $\sigma\left(B_{i}\right)=0$, each vertex $v$ with $\beta(v)=B_{i}$ is flagged as dominated, i. e., $v \in V_{i-1}^{S}$. Thus, by setting $S_{i}=S_{i-1}$ (line 11) and $D_{i}=D_{i-1}$, all three statements are satisfied for $i$. Next, consider the case when $\sigma\left(B_{i}\right)>0$. Therefore, $G$ contains a vertex $u$ with $\beta(u)=B_{i}$ and $u \notin V_{i-1}^{S}$. Then, the algorithm sets $S_{i}=S_{i-1} \cup\left\{c\left(B_{i}\right)\right\}$ and flags all such $u$ as dominated (see line 7 to line 9). Thus, $u \in V_{i}^{S}$ and statement (iii) is satisfied. Let $d_{u}$ be a vertex in $D$ with minimal distance to $u$. Thus, $d_{G}\left(d_{u}, u\right) \leq r(u)$, i. e., $d_{u}$ is in the $r$-neighbourhood of $u$. Note that, because $u \notin V_{i-1}^{S}$ and $V_{i-1}^{D} \subseteq V_{i-1}^{S}, d_{u} \notin D_{i-1}$. Therefore, by setting $D_{i}=D_{i-1} \cup\left\{d_{u}\right\},\left|S_{i}\right|=\left|S_{i-1}\right|+1=\left|D_{i-1}\right|+1=\left|D_{i}\right|$ and statement (ii) is satisfied. Recall that $\beta(u)$ points to the bag closest to the root of $T_{r}$ which intersects the $r$-neighbourhood of $u$. Thus, because $\beta(u)=B_{i}$, each bag $B \neq B_{i}$ with $d_{G}(u, B) \leq r(u)$ is a descendant of $B_{i}$. Therefore, $d_{u}$ is in $B_{i}$ or in a descendant of $B_{i}$. Let $v$ be an arbitrary vertex of $G$ such that $v \notin V_{i-1}^{S}$ and $d_{G}\left(v, d_{u}\right) \leq r(v)$, i. e., $v$ is dominated by $d_{u}$ but not by $S_{i-1}$. Due to statement (iii) of the induction hypothesis, $\beta(v)=B_{j}$ with $j \geq i$, i. e., $B_{j}$ cannot be a descendant of $B_{i}$. Partition the vertices of $G$ into the sets $V_{i}^{\uparrow}$ and $V_{i}^{\downarrow}$ such that $V_{i}^{\downarrow}$ contains the vertices which are contained in $B_{i}$ or in a descendant of $B_{i}$. If $v \in V_{i}^{\downarrow}$, then there is a path of length at most $r(v)$ from $v$ to $B_{j}$ passing through $B_{i}$. If $v \in V_{i}^{\uparrow}$, then, because $d_{u} \in V_{i}^{\downarrow}$, there is a path of length at most $r(v)$ from $v$ to $d_{u}$ passing through $B_{i}$. Therefore, $d_{G}\left(v, B_{i}\right) \leq r(v)$. That is, each vertex $r$-dominated by $d_{u}$, is $(r+\rho)$-dominated by some $c\left(B_{j}\right) \in S_{i}$. Therefore, because $S_{i}=S_{i-1} \cup\left\{c\left(B_{i}\right)\right\}$ and $D_{i}=D_{i-1} \cup\left\{d_{u}\right\}, v \in V_{i}^{S} \cap V_{i}^{D}$ and, thus, statement (i) is satisfied.

Proof (Complexity). Computing $T_{r}$ (line 1) takes at most $\mathcal{O}(n m)$ time (see Lemma 10p. Because $T_{r}$ has at most $n$ bags, computing a BFS-order of $T_{r}$ (line 4p takes at most $\mathcal{O}(n)$ time. For some bag $B_{i}$, determining all vertices $u$ with $d_{G}\left(u, B_{i}\right) \leq r(u)$, flagging $u$ as dominated, and decreasing $\sigma(\beta(u))$ (line 7 to line 9p can be done in $\mathcal{O}(m)$ time by performing a BFS starting at all vertices of $B_{i}$ simultaneously. Therefore, because $T_{r}$ has at most $n$ bags, Algorithm 5 requires at most $\mathcal{O}(\mathrm{nm})$ total time. 


\subsection{Connected $r$-Domination}

In this subsection, we show how to compute a connected $(r+5 \rho)$-dominating set and a connected $(r+3 \lambda)$-dominating set for $G$. For both results, we use almost the same algorithm. To identify and emphasise the differences, we use the label $(\mathcal{})$ for parts which are only relevant to determine a connected $(r+5 \rho)$-dominating set and use the label $(\diamond)$ for parts which are only relevant to determine a connected $(r+3 \lambda)$-dominating set.

For the remainder of this subsection, let $D_{r}$ be a minimum connected $r$ dominating set of $G$. For $(\diamond) \phi=3 \rho$ or $(\diamond) \phi=2 \lambda$, let $T_{\phi}$ be a minimum $(r+\phi)$-covering subtree of $\mathcal{T}$ as computed by Algorithm 4

The idea of our algorithm is to, first, compute $T_{\phi}$ and, second, compute a small enough connected set $C_{\phi}$ such that $C_{\phi}$ intersects each bag of $T_{\phi}$. Lemma 11 below shows that such a set $C_{\phi}$ is an $(r+(\phi+\lambda))$-dominating set.

Lemma 11. Let $C_{\phi}$ be a connected set that contains at least one vertex of each leaf of $T_{\phi}$. Then, $C_{\phi}$ is an $(r+(\phi+\lambda))$-dominating set.

Proof. Clearly, since $C_{\phi}$ is connected and contains a vertex of each leaf of $T_{\phi}$, $C_{\phi}$ contains a vertex of every bag of $T_{\phi}$. By construction of $T_{\phi}$, for each vertex $v$ of $G, T_{\phi}$ contains a bag $B$ such that $d_{G}(v, B) \leq r(v)+\phi$. Therefore, $d_{G}\left(v, C_{\phi}\right) \leq$ $r(v)+\phi+\lambda$, i.e., $C_{\phi}$ is an $(r+(\phi+\lambda))$-dominating set.

To compute a connected set $C_{\phi}$ which intersects all leaves of $T_{\phi}$, we first consider the case when $T_{\rho}$ contains only one bag $B$. In this case, we can construct $C_{\phi}$ by simply picking an arbitrary vertex $v \in B$ and setting $C_{\phi}=\{v\}$. Similarly, if $T_{\rho}$ contains exactly two bags $B$ and $B^{\prime}$, pick a vertex $v \in B \cap B^{\prime}$ and set $C_{\phi}=\{v\}$. In both cases, due to Lemma $11 . C_{\phi}$ is clearly an $(r+(\phi+\lambda))$-dominating set with $\left|C_{\phi}\right| \leq\left|D_{r}\right|$.

Now, consider the case when $T_{\phi}$ contains at least three bags. Additionally, assume that $T_{\phi}$ is a rooted tree such that its root $R$ is a leaf.

Notation. Based on its degree in $T_{\phi}$, we refer to each bag $B$ of $T_{\phi}$ either as leaf, as path bag if $B$ has degree 2, or as branching bag if $B$ has a degree larger than 2 . Additionally, we call a maximal connected set of path bags a path segment of $T_{\phi}$. Let $\mathbb{L}$ denote the set of leaves, $\mathbb{P}$ denote the set of path segments, and $\mathbb{B}$ denote the set of branching bags of $T_{\phi}$. Clearly, for any given tree $T$, the sets $\mathbb{L}, \mathbb{P}$, and $\mathbb{B}$ are pairwise disjoint and can be computed in linear time.

Let $B$ and $B^{\prime}$ be two adjacent bags of $T_{\phi}$ such that $B$ is the parent of $B^{\prime}$. We call $S=B \cap B^{\prime}$ the up-separator of $B^{\prime}$, denoted as $S^{\uparrow}\left(B^{\prime}\right)$, and a down-separator of $B$, denoted as $S^{\downarrow}(B)$, i. e., $S=S^{\uparrow}\left(B^{\prime}\right)=S^{\downarrow}(B)$. Note that a branching bag has multiple down-separators and that (with exception of $R$ ) each bag has exactly one up-separator. For each branching bag $B$, let $\mathcal{S}^{\downarrow}(B)$ be the set of down-separators of $B$. Accordingly, for a path segment $P \in \mathbb{P}, S^{\uparrow}(P)$ is the up-separator of the bag in $P$ closest to the root and $S^{\downarrow}(P)$ is the down separator of the bag in $P$ furthest from the root. Let $\nu$ be a function that assigns a vertex of $G$ to a given separator. Initially, $\nu(S)$ is undefined for each separator $S$. 
Algorithm. Now, we show how to compute $C_{\phi}$. We, first, split $T_{\phi}$ into the sets $\mathbb{L}, \mathbb{P}$, and $\mathbb{B}$. Second, for each $P \in \mathbb{P}$, we create a small connected set $C_{P}$, and, third, for each $B \in \mathbb{B}$, we create a small connected set $C_{B}$. If this is done properly, the union $C_{\phi}$ of all these sets forms a connect set which intersects each bag of $T_{\phi}$.

Note that, due to properties of tree-decompositions, it can be the case that there are two bags $B$ and $B^{\prime}$ which have a common vertex $v$, even if $B$ and $B^{\prime}$ are non-adjacent in $T_{\phi}$. In such a case, either $v \in S^{\downarrow}(B) \cap S^{\uparrow}\left(B^{\prime}\right)$ if $B$ is an ancestor of $B^{\prime}$, or $v \in S^{\uparrow}(B) \cap S^{\uparrow}\left(B^{\prime}\right)$ if neither is ancestor of the other. To avoid problems caused by this phenomena and to avoid counting vertices multiple times, we consider any vertex in an up-separator as part of the bag above. That is, whenever we process some segment or bag $X \in \mathbb{L} \cup \mathbb{P} \cup \mathbb{B}$, even though we add a vertex $v \in S^{\uparrow}(X)$ to $C_{\phi}, v$ is not contained in $C_{X}$.

Processing Path Segments. First, after splitting $T_{\phi}$, we create a set $C_{P}$ for each path segment $P \in \mathbb{P}$ as follows. We determine $S^{\uparrow}(P)$ and $S^{\downarrow}(P)$ and then find a shortest path $Q_{P}$ from $S^{\uparrow}(P)$ to $S^{\downarrow}(P)$. Note that $Q_{P}$ contains exactly one vertex from each separator. Let $x \in S^{\uparrow}(P)$ and $y \in S^{\downarrow}(P)$ be these vertices. Then, we set $\nu\left(S^{\uparrow}(P)\right)=x$ and $\nu\left(S^{\downarrow}(P)\right)=y$. Last, we add the vertices of $Q_{P}$ into $C_{\phi}$ and define $C_{P}$ as $Q_{P} \backslash S^{\uparrow}(P)$. Let $C_{\mathbb{P}}$ be the union of all sets $C_{P}$, i. e., $C_{\mathbb{P}}=\bigcup_{P \in \mathbb{P}} C_{P}$.

Lemma 12. $\left|C_{\mathbb{P}}\right| \leq\left|D_{r}\right|-\phi \cdot \Lambda\left(T_{\phi}\right)$.

Proof. Recall that $T_{\phi}$ is a minimum $(r+\phi)$-covering subtree of $\mathcal{T}$. Thus, by Corollary 5, for each leaf $B \in \mathbb{L}$ of $T_{\phi}$, there is a vertex $v$ in $G$ such that $B$ is the only bag of $T_{\phi}$ with $d_{G}(v, B) \leq r(v)+\phi$. Because $D_{r}$ is a connected $r$-dominating set, $D_{r}$ intersects the $r$-neighbourhood of each of these vertices $v$. Thus, by properties of tree-decompositions, $D_{r}$ intersects each bag of $T_{\phi}$. Additionally, for each such $v, D_{r}$ contains a path $D_{v}$ with $\left|D_{v}\right| \geq \phi$ such that $D_{v}$ intersects the $r$-neighbourhood of $v$, intersects the corresponding leaf $B$ of $T_{\phi}$, and does not intersect $S^{\uparrow}(B)\left(S^{\downarrow}(B)\right.$ if $\left.B=R\right)$. Let $D_{\mathbb{L}}$ be the union of all such sets $D_{v}$. Therefore, $\left|D_{\mathbb{L}}\right| \geq \phi \cdot \Lambda\left(T_{\phi}\right)$.

Because $D_{r}$ intersects each bag of $T_{\phi}, D_{r}$ also intersects the up- and downseparators of each path segment. For a path segment $P \in \mathbb{P}$, let $x$ and $y$ be two vertices of $D_{r}$ such that $x \in S^{\uparrow}(P), y \in S^{\downarrow}(P)$, and for which the distance in $G\left[D_{r}\right]$ is minimal. Let $D_{P}$ be the set of vertices on the shortest path in $G\left[D_{r}\right]$ from $x$ to $y$ without $x$, i. e., $x \notin D_{P}$. Note that, by construction, for each $P \in \mathbb{P}, D_{P}$ contains exactly one vertex in $S^{\downarrow}(P)$ and no vertex in $S^{\uparrow}(P)$. Thus, for all $P, P^{\prime} \in \mathbb{P}$, $D_{P} \cap D_{P^{\prime}}=\emptyset$. Let $D_{\mathbb{P}}$ be the union of all such sets $D_{P}$, i. e., $D_{\mathbb{P}}=\bigcup_{P \in \mathbb{P}} D_{P}$. By construction, $\left|D_{\mathbb{P}}\right|=\sum_{P \in \mathbb{P}}\left|D_{P}\right|$ and $D_{\mathbb{L}} \cap D_{\mathbb{P}}=\emptyset$. Therefore, $\left|D_{r}\right| \geq\left|D_{\mathbb{P}}\right|+\left|D_{\mathbb{L}}\right|$ and, hence,

$$
\sum_{P \in \mathbb{P}}\left|D_{P}\right| \leq\left|D_{r}\right|-\left|D_{\mathbb{L}}\right| \leq\left|D_{r}\right|-\phi \cdot \Lambda\left(T_{\phi}\right) .
$$

Recall that, for each $P \in \mathbb{P}$, the sets $C_{P}$ and $D_{P}$ are constructed based on a path from $S^{\uparrow}(P)$ to $S^{\downarrow}(P)$. Since $C_{P}$ is based on a shortest path in $G$, it follows 
that $\left|C_{P}\right|=d_{G}\left(S^{\uparrow}(P), S^{\downarrow}(P)\right) \leq\left|D_{P}\right|$. Therefore,

$$
\left|C_{\mathbb{P}}\right| \leq \sum_{P \in \mathbb{P}}\left|C_{P}\right| \leq \sum_{P \in \mathbb{P}}\left|D_{P}\right| \leq\left|D_{r}\right|-\phi \cdot \Lambda\left(T_{\phi}\right) .
$$

Processing Branching Bags. After processing path segments, we process the branching bags of $T_{\phi}$. Similar to path segments, we have to ensure that all separators are connected. Branching bags, however, have multiple down-separators. To connect all separators of some bag $B$, we pick a vertex $s$ in each separator $S \in \mathcal{S}^{\downarrow}(B) \cup\left\{S^{\uparrow}(B)\right\}$. If $\nu(S)$ is defined, we set $s=\nu(S)$. Otherwise, we pick an arbitrary $s \in S$ and set $\nu(S)=s$. Let $\mathcal{S}^{\downarrow}(B)=\left\{S_{1}, S_{2}, \ldots\right\}, s_{i}=\nu\left(S_{i}\right)$, and $t=\nu\left(S^{\uparrow}(B)\right)$. We then connect these vertices as follows. (See Figure 3 for an illustration.)

$(\varnothing)$ Connect each vertex $s_{i}$ via a shortest path $Q_{i}$ (of length at most $\rho$ ) with the center $c(B)$ of $B$. Additionally, connect $c(B)$ via a shortest path $Q_{t}$ (of length at most $\rho$ ) with $t$. Add all vertices from the paths $Q_{i}$ and from the path $Q_{t}$ into $C_{\phi}$ and let $C_{B}$ be the union of these paths without $t$.

$(\diamond)$ Connect each vertex $s_{i}$ via a shortest path $Q_{i}$ (of length at most $\lambda$ ) with $t$. Add all vertices from the paths $Q_{i}$ into $C_{\phi}$ and let $C_{B}$ be the union of these paths without $t$.

Let $C_{\mathbb{B}}$ be the union of all created sets $C_{B}$, i.e., $C_{\mathbb{B}}=\bigcup_{B \in \mathbb{B}} C_{B}$.

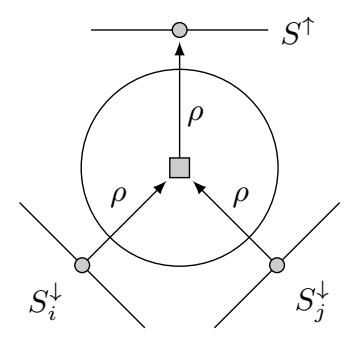

$(\odot)$

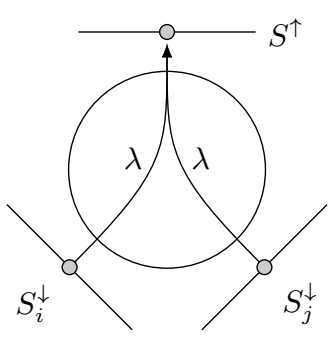

$(\diamond)$

Fig. 3. Construction of the set $C_{B}$ for a branching bag $B$.

Before analysing the cardinality of $C_{\mathbb{B}}$ in Lemma 14 below, we need an axillary lemma.

Lemma 13. For a tree $T$ which is rooted in one of its leaves, let $b$ denote the number of branching nodes, $c$ denote the total number of children of branching nodes, and $l$ denote the number of leaves. Then, $c+b \leq 3 l-1$ and $c \leq 2 l-1$.

Proof. Assume that we construct $T$ by starting with only the root and then step by step adding leaves to it. Let $T_{i}$ be the subtree of $T$ with $i$ nodes during 
this construction. We define $b_{i}, c_{i}$, and $l_{i}$ accordingly. Now, assume by induction over $i$ that Lemma 13 is true for $T_{i}$. Let $v$ be the leaf we add to construct $T_{i+1}$ and let $u$ be its neighbour.

First, consider the case when $u$ is a leaf of $T_{i}$. Then, $u$ becomes a path node of $T_{i+1}$. Therefore, $b_{i+1}=b_{i}, c_{i+1}=c_{i}$, and $l_{i+1}=l_{i}$. Next, assume that $u$ is path node of $T_{i}$. Then, $u$ is a branch node of $T_{i+1}$. Thus, $b_{i+1}=b_{i}+1, c_{i+1}=c_{i}+2$, and $l_{i+1}=l_{i}+1$. Therefore, $c_{i+1}+b_{i+1}=c_{i}+b_{i}+3 \leq 3\left(l_{i}+1\right)-1=3 l_{i+1}-1$ and $c_{i+1}=c_{i}+2 \leq 2\left(l_{i}+1\right)-1=2 l_{i+1}-1$. It remains to check the case when $u$ is a branch node of $T_{i}$. Then, $b_{i+1}=b_{i}, c_{i+1}=c_{i}+1$, and $l_{i+1}=l_{i}+1$. Thus, $c_{i+1}+b_{i+1}=c_{i}+b_{i}+1 \leq 3 l_{i}-1+1 \leq 3 l_{i+1}-1$ and $c_{i+1}=c_{i}+1 \leq 2 l_{i}-1+1 \leq$ $2 l_{i+1}-1$. Therefore, in all three cases, Lemma 13 is true for $T_{i+1}$.

Lemma 14. $\left|C_{\mathbb{B}}\right| \leq \phi \cdot \Lambda\left(T_{\phi}\right)$.

Proof. For some branching bag $B \in \mathbb{B}$, the set $C_{B}$ contains $(\varnothing)$ a path of length at most $\rho$ for each $S_{i} \in \mathcal{S}^{\downarrow}(B)$ and a path of length at most $\rho$ to $S^{\uparrow}(B)$, or $(\diamond)$ a path of length at most $\lambda$ for each $S_{i} \in \mathcal{S}^{\downarrow}(B)$. Thus, (ऽ) $\left|C_{B}\right| \leq \rho \cdot\left|\mathcal{S}^{\downarrow}(B)\right|+\rho$ or $(\diamond)\left|C_{B}\right| \leq \lambda \cdot\left|\mathcal{S}^{\downarrow}(B)\right|$. Recall that $\mathcal{S}^{\downarrow}(B)$ contains exactly one down-separator for each child of $B$ in $T_{\phi}$ and that $C_{\mathbb{B}}$ is the union of all sets $C_{B}$. Therefore, Lemma 13 implies the following.

$$
\begin{aligned}
\left|C_{\mathbb{B}}\right| & \leq \sum_{B \in \mathbb{B}}\left|C_{B}\right| \\
(\diamond) & \leq \rho \cdot \sum_{B \in \mathbb{B}}\left|\mathcal{S}^{\downarrow}(B)\right|+\rho \cdot|\mathbb{B}| \leq 3 \rho \cdot \Lambda\left(T_{\phi}\right)-1 \\
(\diamond) & \leq \lambda \cdot \sum_{B \in \mathbb{B}}\left|\mathcal{S}^{\downarrow}(B)\right| \quad \leq 2 \lambda \cdot \Lambda\left(T_{\phi}\right)-1 \\
& \leq \phi \cdot \Lambda\left(T_{\phi}\right)-1 .
\end{aligned}
$$

Properties of $C_{\phi}$. We now analyse the created set $C_{\phi}$ and show that $C_{\phi}$ is a connected $(r+\phi)$-dominating set for $G$.

Lemma 15. $C_{\phi}$ contains a vertex in each bag of $T_{\phi}$.

Proof. Clearly, by construction, $C_{\phi}$ contains a vertex in each path bag and in each branching bag. Now, consider a leaf $L$ of $T_{\phi} . L$ is adjacent to a path segment or branching bag $X \in \mathbb{P} \cap \mathbb{B}$. Whenever such an $X$ is processed, the algorithm ensures that all separators of $X$ contain a vertex of $C_{\phi}$. Since one of these separators is also the separator of $L$, it follows that each leaf $L$ and, thus, each bag of $T_{\phi}$ contains a vertex of $C_{\phi}$.

Lemma 16. $\left|C_{\phi}\right| \leq\left|D_{r}\right|$.

Proof. Note that, for each vertex $u$ we add to $C_{\phi}$, we also add $u$ to a unique set $C_{X}$ for some $X \in \mathbb{P} \cap \mathbb{B}$. The exception is the vertex $v$ in $S^{\downarrow}(R)$ which is added to no such set $C_{X}$. It follows from our construction of the sets $C_{X}$ that there 
is only one such vertex $v$ and that $v=\nu\left(S^{\downarrow}(R)\right)$. Thus, $\left|C_{\phi}\right|=\left|C_{\mathbb{P}}\right|+\left|C_{\mathbb{B}}\right|+1$. Now, it follows from Lemma 12 and Lemma 14 that

$$
\left|C_{\phi}\right| \leq\left|D_{r}\right|-\phi \cdot \Lambda\left(T_{\phi}\right)+\phi \cdot \Lambda\left(T_{\phi}\right)-1+1 \leq\left|D_{r}\right| .
$$

Lemma 17. $C_{\phi}$ is connected.

Proof. First, note that, by maximality, two path segments of $T_{\phi}$ cannot share a common separator. Also, note that, when processing a branching bag $B$, the algorithm first checks if, for any separator $S$ of $B, \nu(S)$ is already defined; if this is the case, it will not be overwritten. Therefore, for each separator $S$ in $T_{\phi}, \nu(S)$ is defined and never overwritten.

Next, consider a path segment or branching bag $X \in \mathbb{P} \cup \mathbb{B}$ and let $S$ and $S^{\prime}$ be two separators of $X$. Whenever such an $X$ is processed, our approach ensures that $C_{\phi}$ connects $\nu(S)$ with $\nu\left(S^{\prime}\right)$. Additionally, observe that, when processing $X$, each vertex added to $C_{\phi}$ is connected via $C_{\phi}$ with $\nu(S)$ for some separator $S$ of $X$.

Thus, for any two separators $S$ and $S^{\prime}$ in $T_{\phi}, C_{\phi}$ connects $\nu(S)$ with $\nu\left(S^{\prime}\right)$ and, additionally, each vertex $v \in C_{\phi}$ is connected via $C_{\phi}$ with $\nu(S)$ for some separator $S$ in $T_{\phi}$. Therefore, $C_{\phi}$ is connected.

From Lemma 15. Lemma 16, Lemma 17, and from applying Lemma 11 it follows:

Corollary 6. $C_{\phi}$ is a connected $(r+(\phi+\lambda))$-dominating set for $G$ with $\left|C_{\phi}\right| \leq$ $\left|D_{r}\right|$.

Implementation. Algorithm 6 below implements our approach described above. This also includes the case when $T_{\phi}$ contains at most two bags.

Theorem 4. Algorithm 6 computes a connected $(r+(\phi+\lambda))$-dominating set $C_{\phi}$ with $\left|C_{\phi}\right| \leq\left|D_{r}\right|$ in $\mathcal{O}(n m)$ time.

Proof. Since Algorithm 6 constructs a set $C_{\phi}$ as described above, its correctness follows from Corollary 6. It remains to show that the algorithm runs in $\mathcal{O}(\mathrm{nm})$ time.

Computing $T_{\phi}$ (line 2) can be done in $\mathcal{O}(n m)$ time (see Lemma 10). Picking a vertex $u$ in the case when $T_{\phi}$ contains at most two bags (line 3 to line 6 can be easily done in $\mathcal{O}(n)$ time. Recall that $T_{\phi}$ has at most $n$ bags. Thus, splitting $T_{\phi}$ in the sets $\mathbb{L}, \mathbb{P}$, and $\mathbb{B}$ can be done in $\mathcal{O}(n)$ time.

Determining all up-separators in $T_{\phi}$ can be done in $\mathcal{O}(M)$ time as follows. Process all bags of $T_{\phi}$ in an order such that a bag is processed before its descendants, e.g., use a preorder or BFS-order. Whenever a bag $B$ is processed, determine a set $S \subseteq B$ of flagged vertices, store $S$ as up-separator of $B$, and, afterwards, flag all vertices in $B$. Clearly, $S$ is empty for the root. Because a bag $B$ is processed before its descendants, all flagged vertices in $B$ also belong to its parent. Thus, by properties of tree-decompositions, these vertices are exactly the vertices in $S^{\uparrow}(B)$. Clearly, processing a single bag $B$ takes at most $\mathcal{O}(|B|)$ 


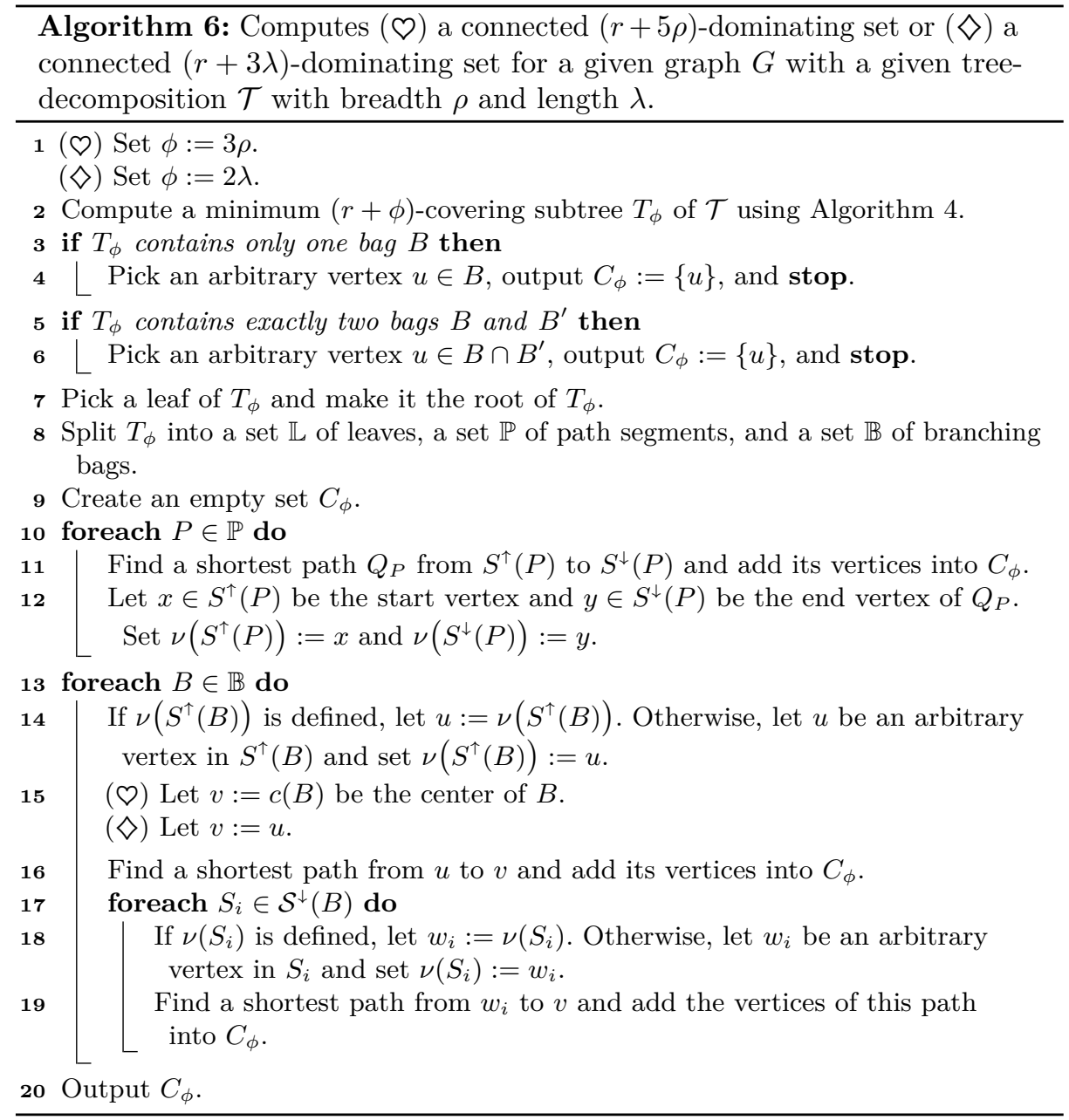

time. Thus, processing all bags takes at most $\mathcal{O}(M)$ time. Note that it is not necessary to determine the down-separators of a (branching) bag. They can easily be accessed via the children of a bag.

Processing a single path segment (line 11 and line 12 can be easily done in $\mathcal{O}(m)$ time. Processing a branching bag $B$ (line 13 to line 19) can be implemented to run in $\mathcal{O}(m)$ time by, first, determining $\nu(S)$ for each separator $S$ of $B$ and, second, running a BFS starting at $v$ (defined in line 15 to connect $v$ with each vertex $\nu(S)$. Because $T_{\phi}$ has at most $n$ bags, it takes at most $\mathcal{O}(n m)$ time to process all path segments and branching bags of $T_{\phi}$.

Therefore, Algorithm 6 runs in $\mathcal{O}(\mathrm{nm})$ total time. 


\section{Implications for the $p$-Center Problem}

The (Connected) $p$-Center problem asks, given a graph $G$ and some integer $p$, for a (connected) vertex set $S$ with $|S| \leq p$ such that $S$ has minimum eccentricity, i. e., there is no (connected) set $S^{\prime}$ with $\operatorname{ecc}_{G}\left(S^{\prime}\right)<\operatorname{ecc}_{G}(S)$. It is known (see, e. g., [3]) that the $p$-Center problem and $r$-Domination problem are closely related. Indeed, one can solve each of these problems by solving the other problem a logarithmic number of times. Lemma 18 below generalises this observation. Informally, it states that we are able to find a $+\phi$-approximation for the $p$-Center problem if we can find a good $(r+\phi)$-dominating set.

Lemma 18. For a given graph $G$, let $D_{r}$ be an optimal (connected) $r$-dominating set and $C_{p}$ be an optimal (connected) p-center. If, for some non-negative integer $\phi$, there is an algorithm to compute a (connected) $(r+\phi)$-dominating set $D$ with $|D| \leq\left|D_{r}\right|$ in $\mathcal{O}(T(G))$ time, then there is an algorithm to compute a (connected) p-center $C$ with $\operatorname{ecc}_{G}(C) \leq \operatorname{ecc}_{G}\left(C_{p}\right)+\phi$ in $\mathcal{O}(T(G) \log n)$ time.

Proof. Let $\mathcal{A}$ be an algorithm which computes a (connected) $(r+\phi)$ dominating set $D=\mathcal{A}(G, r)$ for $G$ with $|D| \leq\left|D_{r}\right|$ in $\mathcal{O}(T(G))$ time. Then we can compute a (connected) $p$-center for $G$ as follows. Make a binary search over the integers $i \in$ $[0, n]$. In each iteration, set $r_{i}(u)=i$ for each vertex $u$ of $G$ and compute the set $D_{i}=\mathcal{A}\left(G, r_{i}\right)$. Then, increase $i$ if $\left|D_{i}\right|>p$ and decrease $i$ otherwise. Note that, by construction, $\operatorname{ecc}_{G}\left(D_{i}\right) \leq i+\phi$. Let $D$ be the resulting set, i. e., out of all computed sets $D_{i}, D$ is the set with minimal $i$ for which $\left|D_{i}\right| \leq p$. It is easy to see that finding $D$ requires at most $\mathcal{O}(T(G) \log n)$ time.

Clearly, $C_{p}$ is a (connected) $r$-dominating set for $G$ when setting $r(u)=$ $\operatorname{ecc}_{G}\left(C_{p}\right)$ for each vertex $u$ of $G$. Thus, for each $i \geq \operatorname{ecc}_{G}\left(C_{p}\right),\left|D_{i}\right| \leq\left|C_{p}\right| \leq p$ and, hence, the binary search decreases $i$ for next iteration. Therefore, there is an $i \leq \operatorname{ecc}_{G}\left(C_{p}\right)$ such that $D=D_{i}$. Hence, $|D| \leq\left|C_{p}\right|$ and $\operatorname{ecc}_{G}(D) \leq$ $\operatorname{ecc}_{G}\left(C_{p}\right)+\phi$.

From Lemma 18 the results in Table 1 and Table 2 follow immediately.

Table 1. Implications of our results for the $p$-Center problem.

\begin{tabular}{lcc}
\hline Approach & Approx. & Time \\
\hline Layering Partition & $+\Delta$ & $\mathcal{O}(m \log n)$ \\
Tree-Decomposition & $+\rho$ & $\mathcal{O}(n m \log n)$ \\
\hline
\end{tabular}

In what follows, we show that, when using a layering partition, we can achieve the results from Table 1 and Table 2 without the logarithmic overhead.

Theorem 5. For a given graph $G, a+\Delta$-approximation for the $p$-Center problem can be computed in linear time. 
Table 2. Implications of our results for the Connected $p$-Center problem.

\begin{tabular}{lcc}
\hline Approach & Approx. & Time \\
\hline Layering Partition & $+2 \Delta$ & $\mathcal{O}(m \alpha(n) \log \Delta \log n)$ \\
Tree-Decomposition & $+\min (5 \rho, 3 \lambda)$ & $\mathcal{O}(n m \log n)$ \\
\hline
\end{tabular}

Proof. First, create a layering partition $\mathcal{T}$ of $G$. Second, find an optimal $p$-center $\mathcal{S}$ for $\mathcal{T}$. Third, create a set $S$ by picking an arbitrary vertex of $G$ from each cluster in $\mathcal{S}$. All three steps can be performed in linear time, including the computation of $\mathcal{S}$ (see [15]).

Let $C$ be an optimal $p$-center for $G$. Note that, by Lemma 1 $C$ also induces a $p$-center for $\mathcal{T}$. Therefore, because $S$ induces an optimal $p$-center for $\mathcal{T}$, Lemma 1 implies that, for each vertex $u$ of $G$,

$$
d_{G}(u, C) \leq d_{G}(u, S) \leq d_{\mathcal{T}}(u, \mathcal{S})+\Delta \leq d_{\mathcal{T}}(u, C)+\Delta \leq d_{G}(u, C)+\Delta .
$$

Theorem 6. For a given graph $G, a+2 \Delta$-approximation for the connected $p$ Center problem can be computed in $\mathcal{O}(m \alpha(n) \log \min (\Delta, p))$ time.

Proof. Recall Algorithm 2 for computing a connected $(r+2 \Delta)$-dominating set. We create Algorithm 2 * by slightly modifying Algorithm 2 as follows. In line 3 , instead of computing an $r$-dominating subtree $T_{r}$ of $\mathcal{T}$, compute an optimal connected $p$-center $T_{p}$ of $\mathcal{T}$ (see [20]). Accordingly, in line 5 compute a $\delta$-dominating subtree of $T_{p}$, check in line 7 if $\left|S_{\delta}\right| \leq\left|T_{p}\right|$ (i. e., if $\left|S_{\delta}\right| \leq p$ ), and output in line 11 the set $S_{\delta}$ with the smallest $\delta$ for which $\left|S_{\delta}\right| \leq p$.

Let $S$ be the set computed by Algorithm $2^{*}$. As shown in the proof of Theorem 2 it follows from Lemma 6 and Corollary 2 that $S$ is connected, $|S| \leq p$, and $S=S_{\delta}$ for some $\delta \leq \Delta$.

Now, let $C$ be an optimal connected $p$-center for $G$. Clearly, by definition of $C$ and by Lemma $11 \operatorname{ecc}_{G}(C) \leq \operatorname{ecc}_{G}\left(S_{\delta}\right) \leq \operatorname{ecc}_{\mathcal{T}}\left(T_{\delta}\right)+\Delta$. Because $T_{\delta}$ is a $\delta$-dominating subtree of $T_{p}$, $\operatorname{ecc}_{\mathcal{T}}\left(T_{\delta}\right) \leq \operatorname{ecc}_{\mathcal{T}}\left(T_{p}\right)+\delta$. Let $T_{C}$ be the subtree of $\mathcal{T}$ induced by $C$, i. e., the subtree of $\mathcal{T}$ induced by the clusters which contain vertices of $C$. Then, because $T_{p}$ is an optimal connected $p$-center for $\mathcal{T}$ and, clearly, $\left|T_{C}\right| \leq p, \operatorname{ecc}_{\mathcal{T}}\left(T_{p}\right) \leq \operatorname{ecc}_{\mathcal{T}}\left(T_{C}\right)$. Therefore, since $\delta \leq \Delta, \operatorname{ecc}_{G}(C) \leq \operatorname{ecc}_{G}\left(S_{\delta}\right) \leq$ $\operatorname{ecc}_{\mathcal{T}}\left(T_{C}\right)+2 \Delta$ and, by Lemma $1 . \operatorname{ecc}_{G}(C) \leq \operatorname{ecc}_{G}\left(S_{\delta}\right) \leq \operatorname{ecc}_{G}(C)+2 \Delta$.

As shown in the proof of Theorem 2, the one-sided binary search of Algorithm $2{ }^{k}$ has at most $\mathcal{O}(\log \Delta)$ iterations. Because $\left|T_{p}\right| \leq p, T_{p}$ contains a cluster with eccentricity at most $\lceil p / 2\rceil$ in $T_{p}$. Therefore, for any $\delta \geq\lceil p / 2\rceil$, $\left|T_{\delta}\right|=\left|S_{\delta}\right|=1$ and, thus, the algorithm decreases $\delta$. Hence, the one-sided binary search of Algorithm $2^{k}$ has at most $\mathcal{O}(\log p)$ iterations. Therefore, the algorithm runs in at most $\mathcal{O}(m \alpha(n) \log \min (\Delta, p))$ total time.

\section{References}

1. Abboud, A., Williams, V.V., Wang, J., Approximation and fixed parameter subquadratic algorithms for radius and diameter in sparse graphs, Proceedings of the 
Twenty-Seventh Annual ACM-SIAM Symposium on Discrete Algorithms, 377-391, 2016.

2. Abu-Ata, M., Dragan, F.F., Metric tree-like structures in real-life networks: an empirical study Networks 67 (1), 49-68, 2016.

3. Brandstädt, A., Chepoi, V., and Dragan, F.F., The Algorithmic Use of Hypertree Structure and Maximum Neighbourhood Orderings. Discrete Applied Mathematics $82(1-3), 43-77,1998$.

4. Brandstädt, A., Chepoi, V., and Dragan, F.F., Distance approximating trees for chordal and dually chordal graphs. Journal of Algorithms 30, 166-184, 1999.

5. Chepoi, V., Dragan, F.F., A note on distance approximating trees in graphs. European Journal of Combinatorics 21, 761-766, 2000.

6. Chepoi, V.D., Dragan, F.F., Estellon, B., Habib, M., Vaxes, Y., Diameters, centers, and approximating trees of $\delta$-hyperbolic geodesic spaces and graphs, Proceedings of the 24th Annual ACM Symposium on Computational Geometry (SoCG 2008), 59-68, 2008.

7. Chepoi, V., Estellon, B., Packing and Covering $\delta$-Hyperbolic Spaces by Balls, Lecture Notes in Computer Science 4627, 59-73, 2007.

8. Chlebík, M., Chlebíková, J., Approximation hardness of dominating set problems in bounded degree graphs, Information and Computation 206, 1264-1275, 2008.

9. Cormen, T.H., Leiserson, C.E., Rivest, R.L., Stein, C., Introduction to Algorithms (3. ed.), MIT Press, 2009.

10. Dourisboure, Y., Dragan, F.F., Gavoille, C., and Yan, C., Spanners for bounded tree-length graphs, Theoretical Computer Science 383 (1), 34-44, 2007.

11. Downey, R.G., Fellows, M.R., Parameterized Complexity, Springer, 1999.

12. Dragan, F.F., HT-graphs: centers, connected $r$-domination and Steiner trees, Computer Science Journal of Moldova 1 (2), 64-83, 1993.

13. Edwards, K., Kennedy, K., Saniee, I., Fast Approximation Algorithms for p-centers in Large $\delta$-hyperbolic Graphs, WAW 2016, Lecture Notes in Computer Science 10088, 60-73, 2016.

14. Escoffier, B., Paschos, V.Th., Completeness in approximation classes beyond APX. Theoretical Computer Science 359 (1-3), 369-377, 2006.

15. Frederickson, G.N.: Parametric Search and Locating Supply Centers in Trees. WADS 1991, Lecture Notes in Computer Science 519, 299-319, 1991.

16. Gonzalez, T., Clustering to minimize the maximum intercluster distance, Theoretical Computer Science, 38, 293-306, 1985.

17. Guha, S., Khuller, S., Approximation algorithms for connected dominating sets, Algorithmica 20 (4), 374-387, 1998.

18. Niedermeier, R., Invitation to fixed-parameter algorithms, Oxford Lecture Series in Mathematics and Its Applications, Oxford University Press, 2006.

19. Raz, R., Safra, S., A sub-constant error-probability low-degree test, and sub-constant error-probability PCP characterization of NP, Proc. 29th Annual ACM Symposium on Theory of Computing, 475-484, 1997.

20. Yen, W.C.-K., Chen, C.-T.: The p-center problem with connectivity constraint. Applied Mathematical Sciences 1 (27), 1311-1324, 2007. 